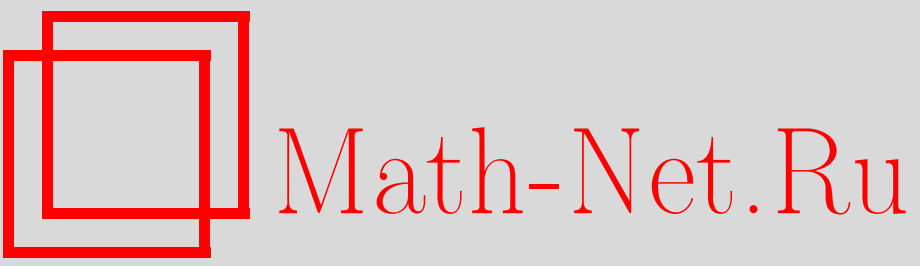

B. Bercu, L. Coutin, N. Savy, Sharp large deviations for the fractional OrnsteinUhlenbeck process, Теория вероятн. и ее примен., 2010, том 55, выпуск 4, 732771

DOI: https://doi.org/10.4213/tvp4280

Использование Общероссийского математического портала Math-Net.Ru подразумевает, что вы прочитали и согласны с пользовательским соглашением http://www.mathnet.ru/rus/agreement

Параметры загрузки:

IP : 54.166 .219 .16

26 апреля 2023 г., 13:46:51 
2010

(c) 2010 г. $\quad$ BERCU B.*, COUTIN L.**, SAVY N.**

\title{
SHARP LARGE DEVIATIONS FOR THE FRACTIONAL ORNSTEIN-UHLENBECK PROCESS
}

\begin{abstract}
Изучаются точные асимптотики в больших уклонениях энергии и оценки максимального правдоподобия для процесса ОрнштейнаУленбека, построенного по фрактальному броуновскому движению с параметром Харста, большим 1/2.

Ключевые слова и фразы: большие уклонения, процесс Орнштейна-Уленбека, фрактальное броуновское движение.
\end{abstract}

1. Introduction. Since the pioneer works of Kolmogorov, Hurst, and Mandelbrot, a wide range of literature is available on the statistical properties of fractional Brownian motion. On the other hand, one can realize that its large deviations properties were not deeply investigated. Our purpose is to establish sharp large deviations for functionals associated with the Ornstein-Uhlenbeck process driven by a fractional Brownian motion

$$
d X_{t}=\theta X_{t} d t+d W_{t}^{H},
$$

where the initial state $X_{0}$ is 0 and the drift parameter $\theta$ is strictly negative. The process $\left(W_{t}^{H}\right)$ is a fractional Brownian motion with the Hurst parameter $0<H<1$, which means that $\left(W_{t}^{H}\right)$ is a Gaussian process with continuous paths such that $W_{0}^{H}=0, \mathbf{E}\left[W_{t}^{H}\right]=0$ and

$$
\mathbf{E}\left[W_{t}^{H} W_{s}^{H}\right]=\frac{1}{2}\left(t^{2 H}+s^{2 H}-|t-s|^{2 H}\right) .
$$

The weighting function $w$ defined, for all $0<s<t$, by $w(t, s)=$ $w_{H}^{-1} s^{-H+1 / 2}(t-s)^{-H+1 / 2}$, where $w_{H}$ is a normalizing positive constant, plays a fundamental role for stochastic calculus associated with $\left(W_{t}^{H}\right)$. In particular, for $t>0$, let

$$
M_{t}=\int_{0}^{t} w(t, s) d W_{s}^{H}
$$

*Université Bordeaux 1, Institut de Mathématiques de Bordeaux, UMR C5251, 351, cours de la Libération, 33405 Talence cedex, France; e-mail: bernard.bercu@math.ubordeaux1.fr

${ }^{* *}$ Université Paul Sabatier, Institut de Mathématiques de Toulouse, UMR C5583, 31062 Toulouse Cedex 09, France; e-mail: laure.coutin@math.univ-toulouse.fr; nicolas.savy@math.univ-toulouse.fr 
It was proved in $\left[14\right.$, p. 578] that $\left(M_{t}\right)$ is a Gaussian martingale with quadratic variation $\langle M\rangle_{t}=\lambda_{H}^{-1} t^{2-2 H}$, where

$$
\lambda_{H}=\frac{2 H \Gamma(3-2 H) \Gamma(H+1 / 2)}{\Gamma(3 / 2-H)}
$$

and $\Gamma$ stands for the classical gamma function. Moreover, it follows from Jost's formula [11] on the transformation of the process $\left(W_{t}^{H}\right)$ into $\left(W_{t}^{1-H}\right)$ that

$$
W_{t}^{H}=\left(\frac{2 H}{\Gamma(2 H) \Gamma(3-2 H)}\right)^{1 / 2} \int_{0}^{t}(t-s)^{2 H-1} d W_{s}^{1-H} .
$$

Consequently, we assume in all the sequel that $1 / 2<H<1$, since our investigation also holds without any problem in the case $0<H<1 / 2$. It is also more convenient to study the behavior of

$$
Y_{t}=\int_{0}^{t} w(t, s) d X_{s}=\theta \int_{0}^{t} w(t, s) X_{s} d s+M_{t} .
$$

It was shown in [12] that relation (1.3) can be rewritten as

$$
Y_{t}=\theta \int_{0}^{t} Q_{s} d\langle M\rangle_{s}+M_{t}
$$

where the process $\left(Q_{t}\right)$ satisfies for all $t>0$

$$
Q_{t}=\frac{l_{H}}{2}\left(t^{2 H-1} Y_{t}+\int_{0}^{t} s^{2 H-1} d Y_{s}\right)
$$

with $l_{H}=\lambda_{H} /(2(1-H))$. It follows from (1.4) that the score function, which is the derivative of the log-likelihood function from observations over the interval $[0, T]$, is given by

$$
\Sigma_{T}(\theta)=\int_{0}^{T} Q_{t} d Y_{t}-\theta \int_{0}^{T} Q_{t}^{2} d\langle M\rangle_{t} .
$$

Via a similar approach as the one of [3] for the Ornstein-Uhlenbeck process, we shall investigate the large deviation properties for random variables associated with (1.1) such as the energy

$$
S_{T}=\int_{0}^{T} Q_{t}^{2} d\langle M\rangle_{t}
$$

as well as the maximum likelihood estimator of $\theta$, solution of $\Sigma_{T}(\theta)=0$, given by

$$
\hat{\theta}_{T}=\frac{\int_{0}^{T} Q_{t} d Y_{t}}{\int_{0}^{T} Q_{t}^{2} d\langle M\rangle_{t}} .
$$


We also wish to mention the recent work [4] concerning the large deviation properties of the log-likelihood ratio

$$
\left(\theta_{0}-\theta_{1}\right) \int_{0}^{T} Q_{t} d Y_{t}-\frac{\theta_{0}^{2}-\theta_{1}^{2}}{2} \int_{0}^{T} Q_{t}^{2} d\langle M\rangle_{t} .
$$

As usual, we say that a family of real random variables $\left(Z_{T}\right)$ satisfies a Large Deviation Principle (LDP) with a rate function $I$, if $I$ is a lower semicontinuous function from $\mathbf{R}$ to $[0,+\infty]$ such that, for any closed set $F \subset \mathbf{R}$,

$$
\limsup _{T \rightarrow \infty} \frac{1}{T} \ln \mathbf{P}\left(Z_{T} \in F\right) \leqslant-\inf _{x \in F} I(x),
$$

while for any open set $G \subset \mathbf{R}$,

$$
-\inf _{x \in G} I(x) \leqslant \liminf _{T \rightarrow \infty} \frac{1}{T} \ln \mathbf{P}\left(Z_{T} \in G\right) .
$$

Moreover, $I$ is a good rate function if its level sets are compact subsets of R. We refer the reader to the excellent book [7] on the theory of large deviations, see also [2], [9]. A classical tool for proving an LDP for $S_{T}$ and $\hat{\theta}_{T}$ is the normalized cumulant generating function

$$
\mathscr{L}_{T}(a, b)=\frac{1}{T} \ln \mathbf{E}\left[\exp \left(\mathscr{Z}_{T}(a, b)\right)\right]
$$

where, for any $(a, b) \in \mathbf{R}^{2}$,

$$
\mathscr{Z}_{T}(a, b)=a \int_{0}^{T} Q_{t} d Y_{t}+b \int_{0}^{T} Q_{t}^{2} d\langle M\rangle_{t} .
$$

The random variable $\mathscr{Z}_{T}(a, b)$ allows us a unified presentation of our results. In order to establish an LDP for $S_{T}$ and $\hat{\theta}_{T}$, it is enough to prove an LDP for $\mathscr{Z}_{T}(0, a)$ and $\mathscr{Z}_{T}(a,-a c)$, respectively. As a matter of fact, we have for all $a, c \geqslant 0$,

$$
\mathbf{P}\left(S_{T} \geqslant c T\right)=\mathbf{P}\left(Z_{T}(0, a) \geqslant a c T\right), \quad \mathbf{P}\left(\hat{\theta}_{T} \geqslant c\right)=\mathbf{P}\left(Z_{T}(a,-a c) \geqslant 0\right) .
$$

The following lemma was partially established by formula (5.12) in [12]. It provides an asymptotic expansion for $\mathscr{L}_{T}$ which enlightens the role of the limit $\mathscr{L}$ of $\mathscr{L}_{T}$ for the LDP, as well as the first order terms $\mathscr{H}$ and $\mathscr{K}_{T}$ for the sharp LDP (in what follows SLDP). One can observe that it is the keystone of all our results.

Lemma 1. Let $\Delta_{H}$ be the effective domain of the limit $\mathscr{L}$ of $\mathscr{L}_{T}$ :

$$
\Delta_{H}=\left\{(a, b) \in \mathbf{R}^{2}: \theta^{2}-2 b>0 \text { and } \sqrt{\theta^{2}-2 b}>\max \left(a+\theta ;-\delta_{H}(a+\theta)\right)\right\},
$$


where $\delta_{H}=(1-\sin (\pi H)) /(1+\sin (\pi H))$. Then, for any $(a, b)$ in the interior of $\Delta_{H}$, if $\varphi(b)=\sqrt{\theta^{2}-2 b}, \tau(a, b)=\varphi(b)-(a+\theta)$, and $r_{T}(b)=$ $r_{H}(\varphi(b) T / 2) \exp (-T \varphi(b))-1$, we have the decomposition

$$
\mathscr{L}_{T}(a, b)=\mathscr{L}(a, b)+\frac{1}{T} \mathscr{H}(a, b)+\frac{1}{T} \mathscr{K}_{T}(a, b)+\frac{1}{T} \mathscr{R}_{T}(a, b),
$$

where

$$
\begin{aligned}
\mathscr{L}(a, b) & =-\frac{1}{2}(a+\theta+\varphi(b)), \\
\mathscr{H}(a, b) & =-\frac{1}{2} \ln \frac{\tau(a, b)}{2 \varphi(b)}, \\
\mathscr{K}_{T}(a, b) & =-\frac{1}{2} \ln \left(1+\frac{2 \varphi(b)-\tau(a, b)}{2 \varphi(b)} r_{T}(b)\right),
\end{aligned}
$$

with the function $r_{H}$ defined for all $z \in \mathbf{C}$, with $|\arg z|<\pi$, by

$$
r_{H}(z)=\frac{\pi z}{\sin (\pi H)}\left(I_{H}(z) I_{1-H}(z)+I_{-H}(z) I_{H-1}(z)\right),
$$

where $I_{H}$ is the modified Bessel function of the first kind [13]. Finally, the remainder is

$$
\mathscr{R}_{T}(a, b)=-\frac{1}{2} \ln \left(1+\frac{(2 \varphi(b)-\tau(a, b))^{2}}{\tau(a, b)\left(2 \varphi(b)+r_{T}(b)(2 \varphi(b)-\tau(a, b))\right)} e^{-2 T \varphi(b)}\right) .
$$

The proof of Lemma 1 is given in Section 5 .

$\mathrm{R}$ e $\mathrm{m}$ a $\mathrm{r} \mathrm{k} 1$. By use of the duplication formula for the gamma function [13], one can realize that if $H=1 / 2$, then $r_{H}(z)=e^{2 z}+e^{-2 z}$ which immediately leads to $r_{T}(b)=e^{-2 T \varphi(b)}$. Consequently, in that particular case, $\mathscr{K}_{T}(a, b)$ as well as $\mathscr{R}_{T}(a, b)$ go exponentially fast to zero and we find again Lemma 2.1 of [3] which is the keystone for all results in [3].

2. The energy. First of all, we shall focus our attention on the energy $\left(S_{T}\right)$. One can observe that the strong law of large numbers as well as the Central Limit Theorem (CLT) for the sequence $\left(S_{T}\right)$ were not previously established in the literature.

Proposition 1. We have

$$
\lim _{T \rightarrow \infty} \frac{S_{T}}{T}=-\frac{1}{2 \theta} \quad \text { a.s. }
$$

Moreover, we also have the CLT

$$
\frac{1}{\sqrt{T}}\left(S_{T}+\frac{T}{2 \theta}\right) \stackrel{\text { Law }}{\longrightarrow} \mathscr{N}\left(0,-\frac{1}{2 \theta^{3}}\right) .
$$


P r o o f. The almost sure convergence (2.1) clearly follows from Theorem 2 below together with the Borel-Cantelli lemma. As a matter of fact, the sequence $\left(S_{T} / T\right)$ satisfies a SLDP with good rate function $I$ given by $(2.11)$ and $I(c)=0$ if and only if $c=-1 /(2 \theta)$. In order to prove the CLT given by $(2.2)$, denote

$$
V_{T}=\frac{1}{\sqrt{T}}\left(S_{T}+\frac{T}{2 \theta}\right) .
$$

For all $a \in \mathbf{R}$, let $\Lambda_{T}(a)=\mathbf{E}\left[\exp \left(a V_{T}\right)\right]$. We clearly have

$$
\Lambda_{T}(a)=\exp \left(\frac{a \sqrt{T}}{2 \theta}\right) \mathbf{E}\left[\exp \left(\frac{a S_{T}}{\sqrt{T}}\right)\right]
$$

Hence, we deduce from the decomposition (1.9) with $a=0$ and $b=a$ that

$$
\begin{aligned}
\Lambda_{T}(a)=\exp ( & \frac{a \sqrt{T}}{2 \theta}+T \mathscr{L}\left(0, \frac{a}{\sqrt{T}}\right)+\mathscr{H}\left(0, \frac{a}{\sqrt{T}}\right) \\
& \left.+\mathscr{K}_{T}\left(0, \frac{a}{\sqrt{T}}\right)+\mathscr{R}_{T}\left(0, \frac{a}{\sqrt{T}}\right)\right) .
\end{aligned}
$$

On the one hand,

$$
\mathscr{L}\left(0, \frac{a}{\sqrt{T}}\right)=-\frac{1}{2}\left(\theta+\varphi_{T}\right)
$$

where

$$
\varphi_{T}=\sqrt{\theta^{2}-\frac{2 a}{\sqrt{T}}}=-\theta \sqrt{1-\frac{2 a}{\theta^{2} \sqrt{T}}} .
$$

Consequently, since

$$
\varphi_{T}=-\theta+\frac{a}{\theta \sqrt{T}}+\frac{a^{2}}{2 \theta^{3} T}+o\left(\frac{1}{T}\right),
$$

we obtain that

$$
\lim _{T \rightarrow \infty} \frac{a \sqrt{T}}{2 \theta}+T \mathscr{L}\left(0, \frac{a}{\sqrt{T}}\right)=-\frac{a^{2}}{4 \theta^{3}} .
$$

On the other hand, since $\left(\varphi_{T}\right)$ converges to $-\theta$, one can check that

$$
\lim _{T \rightarrow \infty}\left(\mathscr{H}\left(0, \frac{a}{\sqrt{T}}\right)+\mathscr{K}_{T}\left(0, \frac{a}{\sqrt{T}}\right)+\mathscr{R}_{T}\left(0, \frac{a}{\sqrt{T}}\right)\right)=0 .
$$

Therefore, we infer from (2.3)-(2.5) that

$$
\lim _{T \rightarrow \infty} \Lambda_{T}(a)=\exp \left(-\frac{a^{2}}{4 \theta^{3}}\right) .
$$

Convergence (2.6) holds for all $a$ in a neighborhood of the origin which leads to (2.2) and completes the proof of Proposition 1. 
In order to obtain the large deviation properties for $\left(S_{T}\right)$, we shall make use of Lemma 1 with $a=0$ and $b=a$. On the one hand, let

$$
D_{H}=\left\{a \in \mathbf{R}: \theta^{2}-2 a>0 \text { and } \sqrt{\theta^{2}-2 a}>-\delta_{H} \theta\right\} .
$$

It is not hard to see that $\left.D_{H}=\right]-\infty, a_{H}[$, where

$$
a_{H}=\frac{\theta^{2}}{2}\left(1-\delta_{H}^{2}\right) .
$$

Consequently, since $\left|\delta_{H}\right|<1$, one can observe that the origin always belongs to the interior of $D_{H}$. On the other hand, for all $a \in D_{H}$, let $\varphi(a)=$ $\sqrt{\theta^{2}-2 a}$,

$$
\begin{aligned}
L(a) & =\mathscr{L}(0, a)=-\frac{1}{2}\left(\theta+\sqrt{\theta^{2}-2 a}\right), \\
H(a) & =\mathscr{H}(0, a)=-\frac{1}{2} \ln \frac{\varphi(a)-\theta}{2 \varphi(a)}, \\
K_{T}(a) & =\mathscr{K}_{T}(0, a)=-\frac{1}{2} \ln \left(1+\frac{\varphi(a)+\theta}{2 \varphi(a)} r_{T}(a)\right) .
\end{aligned}
$$

The main difficulty comparing to [3] is that the function $L$ is not steep. Actually,

$$
L^{\prime}(a)=\frac{1}{2 \sqrt{\theta^{2}-2 a}},
$$

which implies that $L^{\prime}\left(a_{H}\right)=-1 /\left(2 \theta \delta_{H}\right)$. Moreover, for all $c>0, L^{\prime}(a)=c$ if and only if $a=a_{c}$ with $a_{c}=\left(4 \theta^{2} c^{2}-1\right) /\left(8 c^{2}\right)$. Hence, $a_{c}<a_{H}$ whenever $0<c<-1 /\left(2 \theta \delta_{H}\right)$. Denote by $I$ the Fenchel-Legendre transform of the function $L$. Our first result on the large deviation properties of $\left(S_{T} / T\right)$ is as follows.

Theorem 1. The sequence $\left(S_{T} / T\right)$ satisfies an LDP with good rate function

$$
I(c)= \begin{cases}\frac{(2 \theta c+1)^{2}}{8 c} & \text { if } 0<c \leqslant-\frac{1}{2 \theta \delta_{H}}, \\ \frac{c \theta^{2}}{2}\left(1-\delta_{H}^{2}\right)+\frac{\theta}{2}\left(1-\delta_{H}\right) & \text { if } c \geqslant-\frac{1}{2 \theta \delta_{H}} \\ +\infty & \text { otherwise. }\end{cases}
$$

R e mark 2. If $H=1 / 2$, then $\delta_{H}=0$ and the LDP for $\left(S_{T} / T\right)$ is exactly the one established in [6] for general centered Gaussian processes.

We are now going to improve this result by a SLDP for $\left(S_{T} / T\right)$ inspired by the well-known Bahadur-Rao theorem [1] on the sample mean. 
Theorem 2. The sequence $\left(S_{T} / T\right)$ satisfies an $S L D P$ associated with $L, H$, and $K_{T}$ given by (2.8), (2.9), and (2.10), respectively.

a) For all $-1 /(2 \theta)<c<-1 /\left(2 \theta \delta_{H}\right)$, there exists a sequence $\left(b_{c, k}^{H}\right)$ such that, for any $p>0$ and $T$ large enough,

$$
\mathbf{P}\left(S_{T} \geqslant c T\right)=\frac{\exp \left(-T I(c)+J(c)+K_{H}(c)\right)}{a_{c} \sigma_{c} \sqrt{2 \pi T}}\left[1+\sum_{k=1}^{p} \frac{b_{c, k}^{H}}{T^{k}}+\mathscr{O}\left(\frac{1}{T^{p+1}}\right)\right]
$$

while, for $0<c<-1 /(2 \theta)$,

$$
\mathbf{P}\left(S_{T} \leqslant c T\right)=-\frac{\exp \left(-T I(c)+J(c)+K_{H}(c)\right)}{a_{c} \sigma_{c} \sqrt{2 \pi T}}\left[1+\sum_{k=1}^{p} \frac{b_{c, k}^{H}}{T^{k}}+\mathscr{O}\left(\frac{1}{T^{p+1}}\right)\right],
$$

where

$$
J(c)=-\frac{1}{2} \ln \frac{1-2 \theta c}{2}, \quad K_{H}(c)=-\frac{1}{2} \ln \frac{(1+\sin (\pi H))\left(1+2 \theta c \delta_{H}\right)}{2 \sin (\pi H)},
$$

with

$$
a_{c}=\frac{4 \theta^{2} c^{2}-1}{8 c^{2}}, \quad \sigma_{c}^{2}=4 c^{3} .
$$

Moreover, the coefficients $b_{c, 1}^{H}, \ldots, b_{c, p}^{H}$ can be calculated explicitly as functions of the derivatives of $L$ and $H$ evaluated at point $a_{c}$. They also depend on the Taylor expansion of $K_{T}$ and its derivatives at $a_{c}$.

b) For all $c>-1 /\left(2 \theta \delta_{H}\right)$, there exists a sequence $\left(d_{c, k}^{H}\right)$ such that, for any $p>0$ and $T$ large enough,

$$
\mathbf{P}\left(S_{T} \geqslant c T\right)=\frac{\exp \left(-T I(c)+P_{H}(c)+Q_{H}(c)\right)}{a_{H} \sigma_{H} \sqrt{2 \pi T}}\left[1+\sum_{k=1}^{p} \frac{d_{c, k}^{H}}{T^{k}}+\mathscr{O}\left(\frac{1}{T^{p+1}}\right)\right],
$$

where

$$
P_{H}(c)=-\frac{1}{2} \ln \frac{-\left(1+2 \theta c \delta_{H}\right)}{4 \delta_{H} \sin (\pi H)}, \quad Q_{H}(c)=\frac{(2 H-1)^{2} \sin (\pi H)\left(1+2 \theta c \delta_{H}\right)}{2\left(1-(\sin (\pi H))^{2}\right)}
$$

with

$$
a_{H}=\frac{\theta^{2}\left(1-\delta_{H}^{2}\right)}{2}, \quad \sigma_{H}^{2}=-\frac{1}{2 \theta^{3} \delta_{H}^{3}} .
$$

Moreover, the coefficients $d_{c, 1}^{H}, \ldots, d_{c, p}^{H}$ can be calculated explicitly as functions of the derivatives of $L$ and $H$ evaluated at point $a_{H}$. They also depend on the Taylor expansion of $K_{T}$ and its derivatives at $a_{H}$.

$\mathrm{R}$ e $\mathrm{m}$ a r k 3. For example, the first coefficient $b_{c, 1}^{H}$ is given by

$$
b_{c, 1}^{H}=\frac{1}{\sigma_{c}^{2}}\left(\frac{s_{1}}{a_{c}}-\frac{s_{1}^{2}}{2}-\frac{s_{2}}{2}-\frac{s_{3}}{2 a_{c} \sigma_{c}^{2}}+\frac{s_{1} \ell_{3}}{2 \sigma_{c}^{2}}-\frac{5 \ell_{3}^{2}}{24 \sigma_{c}^{4}}+\frac{\ell_{4}}{8 \sigma_{c}^{2}}-\frac{1}{a_{c}^{2}}\right)+k_{c, 1}^{H},
$$


where $\ell_{q}=L^{(q)}\left(a_{c}\right), h_{q}=H^{(q)}\left(a_{c}\right), s_{q}=h_{q}+k_{q}$, with

$$
\begin{aligned}
k_{1} & =\lim _{T \rightarrow \infty} K_{T}^{\prime}\left(a_{c}\right)=\frac{-4 \theta p_{H} c^{3}}{2+p_{H}(1+2 \theta c)}, \\
k_{2} & =\lim _{T \rightarrow \infty} K_{T}^{\prime \prime}\left(a_{c}\right)=\frac{16 \theta p_{H} c^{5}\left(6+p_{H}(3+2 \theta c)\right)}{\left(2+p_{H}(1+2 \theta c)\right)^{2}}, \\
k_{c, 1}^{H} & =\lim _{T \rightarrow \infty} T\left(K_{T}\left(a_{c}\right)-K_{H}(c)\right)=\frac{c(1+2 \theta c)(2 H-1)^{2}}{2 \sin (\pi H)\left(2+p_{H}(1+2 \theta c)\right)},
\end{aligned}
$$

$p_{H}=(1-\sin (\pi H)) / \sin (\pi H)$. In addition, one can also observe that $\sigma_{c}^{2}=$ $L^{\prime \prime}\left(a_{c}\right)$ and $\sigma_{H}^{2}=L^{\prime \prime}\left(a_{H}\right)$.

Theorem 3. For $c=-1 /\left(2 \theta \delta_{H}\right)$, there exists a sequence $\left(d_{k}^{H}\right)$ such that, for any $p>0$ and $T$ large enough,

$$
\mathbf{P}\left(S_{T} \geqslant c T\right)=\frac{\exp \left(-T I(c)+K_{H}\right) \Gamma(1 / 4)}{2 \pi a_{H} \sigma_{H} T^{1 / 4}}\left[1+\sum_{k=1}^{2 p} \frac{d_{k}^{H}}{(\sqrt{T})^{k}}+\mathscr{O}\left(\frac{1}{T^{p} \sqrt{T}}\right)\right]
$$

where $a_{H}$ and $\sigma_{H}^{2}$ are given by (2.18) and

$$
K_{H}=\frac{1}{2} \ln \left(\delta_{H} \sin (\pi H)\right)+\frac{1}{4} \ln \left(-\theta \delta_{H}\right) .
$$

As before, the coefficients $d_{1}^{H}, \ldots, d_{2 p}^{H}$ may be calculated explicitly.

The proofs of Theorems 2 and 3 are given in Section 4.

3. The maximum likelihood estimator. Our purpose is now to establish similar results for the maximum likelihood estimator $\hat{\theta}_{T}$ of $\theta$. An alternative estimator of $\theta$ may be found in [10]. The almost sure convergence of $\hat{\theta}_{T}$ towards $\theta$ was already proved in [12], see also [15], [16] for related results. We just learned that the CLT was established via a different approach in $[5]$.

Proposition 2. We have

$$
\lim _{T \rightarrow \infty} \hat{\theta}_{T}=\theta \quad \text { a.s. }
$$

Moreover, we also have the CLT

$$
\sqrt{T}\left(\hat{\theta}_{T}-\theta\right) \stackrel{\text { Law }}{\longrightarrow} \mathscr{N}(0,-2 \theta) .
$$

P r o o f. The almost sure convergence (3.1) is given in [12, Proposition 2.2]. For all $c \in \mathbf{R}$, denote

$$
V_{T}(c)=\frac{1}{\sqrt{T}} \int_{0}^{T} Q_{t} d Y_{t}-\left(\frac{c}{\sqrt{T}}+\theta\right) \frac{S_{T}}{\sqrt{T}} .
$$


One can easily check that

$$
\mathbf{P}\left(\sqrt{T}\left(\hat{\theta}_{T}-\theta\right) \leqslant c\right)=\mathbf{P}\left(V_{T}(c) \leqslant 0\right) .
$$

Consequently, in order to prove the CLT given by (3.2), it is only necessary to establish the asymptotic behavior of the sequence $\left(V_{T}(c)\right)$, where the threshold $c$ can be seen as a parameter. For all $(a, c) \in \mathbf{R}^{2}$, let $\Lambda_{T}(a, c)=$ $\mathbf{E}\left[\exp \left(a V_{T}(c)\right)\right]$. We clearly have

$$
\Lambda_{T}(a, c)=\exp \left(T L_{T}\left(\frac{a}{\sqrt{T}}, c_{T}\right)\right), \quad \text { where } c_{T}=-\frac{a}{\sqrt{T}}\left(\frac{c}{\sqrt{T}}+\theta\right) .
$$

Thus, it follows from the decomposition (1.9) that

$$
\begin{aligned}
\Lambda_{T}(a, c)=\exp ( & T \mathscr{L}\left(\frac{a}{\sqrt{T}}, c_{T}\right)+\mathscr{H}\left(\frac{a}{\sqrt{T}}, c_{T}\right) \\
& \left.+\mathscr{K}_{T}\left(\frac{a}{\sqrt{T}}, c_{T}\right)+\mathscr{R}_{T}\left(\frac{a}{\sqrt{T}}, c_{T}\right)\right) .
\end{aligned}
$$

On the one hand,

$$
\mathscr{L}\left(\frac{a}{\sqrt{T}}, c_{T}\right)=-\frac{1}{2}\left(\frac{a}{\sqrt{T}}+\theta+\varphi_{T}\right),
$$

where $\varphi_{T}=\sqrt{\theta^{2}-2 c_{T}}=-\theta \sqrt{1-2 c_{T} / \theta^{2}}$. Hence, since $\varphi_{T}=-\theta-a / \sqrt{T}+$ $\left(a^{2}-2 a c\right) /(2 \theta T)+o(1 / T)$, we deduce that

$$
\lim _{T \rightarrow \infty} T \mathscr{L}\left(\frac{a}{\sqrt{T}}, c_{T}\right)=-\frac{a^{2}-2 a c}{4 \theta} .
$$

On the other hand, since $\left(\varphi_{T}\right)$ converges to $-\theta$, it is not hard to see that

$$
\lim _{T \rightarrow \infty}\left(\mathscr{H}\left(\frac{a}{\sqrt{T}}, c_{T}\right)+\mathscr{K}_{T}\left(\frac{a}{\sqrt{T}}, c_{T}\right)+\mathscr{R}_{T}\left(\frac{a}{\sqrt{T}}, c_{T}\right)\right)=0
$$

The conjunction of (3.4), (3.5), and (3.6) leads to

$$
\lim _{T \rightarrow \infty} \Lambda_{T}(a, c)=\exp \left(-\frac{a^{2}-2 a c}{4 \theta}\right) .
$$

This convergence holds for all $a$ in a neighborhood of the origin. Consequently,

$$
V_{T}(c) \stackrel{\text { Law }}{\longrightarrow} \mathscr{N}\left(\frac{c}{2 \theta}, \frac{-1}{2 \theta}\right) .
$$

Denote by $V(c)$ the limiting distribution of $\left(V_{T}(c)\right)$. It follows from a standard Gaussian calculation that

$$
\mathbf{P}(V(c) \leqslant 0)=\frac{1}{-4 \pi \theta} \int_{-\infty}^{c} \exp \left(\frac{x^{2}}{2 \theta}\right) d x .
$$


Finally, (3.3) and (3.9) imply (3.2) which completes the proof of Proposition 2 .

In order to establish the large deviation properties of $\left(\hat{\theta}_{T}\right)$, we shall make use of the auxiliary random variable defined for all $c \in \mathbf{R}$ by

$$
Z_{T}(c)=\int_{0}^{T} Q_{t} d Y_{t}-c \int_{0}^{T} Q_{t}^{2} d\langle M\rangle_{t}
$$

where we recall that $\mathbf{P}\left(\hat{\theta}_{T} \geqslant c\right)=\mathbf{P}\left(Z_{T}(c) \geqslant 0\right)$. Let

$$
D_{H}=\left\{a \in \mathbf{R}: \theta^{2}+2 a c>0 \text { and } \sqrt{\theta^{2}+2 a c}>\max \left(a+\theta ;-\delta_{H}(a+\theta)\right)\right\} .
$$

After some straightforward calculations, it is not hard to see that

$$
D_{H}= \begin{cases}] a_{1}^{H}, a_{2}^{H}[ & \text { if } c \leqslant \frac{\theta}{2}, \\ ] a_{1}^{H}, a^{c}[ & \text { if } c>\frac{\theta}{2},\end{cases}
$$

where $a^{c}=2(c-\theta)$ and

$$
\begin{aligned}
& a_{1}^{H}=\frac{c-\theta \mu_{H}-\sqrt{c^{2}-2 \theta c \mu_{H}+\theta^{2} \mu_{H}}}{\mu_{H}}, \\
& a_{2}^{H}=\frac{c-\theta \mu_{H}+\sqrt{c^{2}-2 \theta c \mu_{H}+\theta^{2} \mu_{H}}}{\mu_{H}},
\end{aligned}
$$

with $\mu_{H}=\delta_{H}^{2}$. In addition, for all $a \in D_{H}$, let $\varphi(a)=\sqrt{\theta^{2}+2 a c}$ and

$$
\begin{aligned}
L(a) & =\mathscr{L}(a,-c a)=-\frac{1}{2}\left(a+\theta+\sqrt{\theta^{2}+2 a c}\right), \\
H(a) & =\mathscr{H}(a,-c a)=-\frac{1}{2} \ln \frac{\varphi(a)-a-\theta}{2 \varphi(a)}, \\
K_{T}(a) & =\mathscr{K}_{T}(a,-c a)=-\frac{1}{2} \ln \left(1+\frac{\varphi(a)+a+\theta}{2 \varphi(a)} r_{T}(-a c)\right) .
\end{aligned}
$$

The function $L$ is not steep, since the derivative of $L$ is finite at the boundary of $D_{H}$. Moreover, $L^{\prime}(a)=0$ if and only if $a=a_{c}$ with $a_{c}=$ $\left(c^{2}-\theta^{2}\right) /(2 c)$ and $a_{c} \in D_{H}$ whenever $c<\theta / 3$.

Theorem 4. The maximum likelihood estimator $\left(\hat{\theta}_{T}\right)$ satisfies an LDP with good rate function

$$
I(c)= \begin{cases}-\frac{(c-\theta)^{2}}{4 c} & \text { if } c<\frac{\theta}{3}, \\ 2 c-\theta & \text { if } c \geqslant \frac{\theta}{3} .\end{cases}
$$


$\mathrm{R} \mathrm{e} \mathrm{m}$ a r k 4 . One can observe that the rate function $I$ is totally free of the parameter $H$. Hence, $\left(\hat{\theta}_{T}\right)$ shares the same LDP as the one established in [8] for the standard Ornstein-Uhlenbeck process with $H=1 / 2$.

Theorem 5. The maximum likelihood estimator $\left(\hat{\theta}_{T}\right)$ satisfies an SLDP associated with $L, H$, and $K_{T}$ given by (3.11), (3.12), and (3.13), respectively.

a) For all $\theta<c<\theta / 3$, there exists a sequence $\left(b_{c, k}^{H}\right)$ such that, for any $p>0$ and $T$ large enough,

$$
\mathbf{P}\left(\hat{\theta}_{T} \geqslant c\right)=\frac{\exp \left(-T I(c)+J(c)+K_{H}(c)\right)}{\sigma_{c} a_{c} \sqrt{2 \pi T}}\left[1+\sum_{k=1}^{p} \frac{b_{c, k}^{H}}{T^{k}}+\mathscr{O}\left(\frac{1}{T^{p+1}}\right)\right]
$$

while, for $c<\theta$,

$$
\mathbf{P}\left(\hat{\theta}_{T} \leqslant c\right)=-\frac{\exp \left(-T I(c)+J(c)+K_{H}(c)\right)}{\sigma_{c} a_{c} \sqrt{2 \pi T}}\left[1+\sum_{k=1}^{p} \frac{b_{c, k}^{H}}{T^{k}}+\mathscr{O}\left(\frac{1}{T^{p+1}}\right)\right],
$$

where

$$
J(c)=-\frac{1}{2} \ln \frac{(c+\theta)(3 c-\theta)}{4 c^{2}}, \quad K_{H}(c)=-\frac{1}{2} \ln \left(1+p_{H} \frac{(c-\theta)^{2}}{4 c^{2}}\right)
$$

with $p_{H}=(1-\sin (\pi H)) / \sin (\pi H)$,

$$
a_{c}=\frac{c^{2}-\theta^{2}}{2 c}, \quad \sigma_{c}^{2}=-\frac{1}{2 c} .
$$

Moreover, the coefficients $b_{c, 1}^{H}, \ldots, b_{c, p}^{H}$ can be calculated explicitly as in Theorem 2.

b) For all $c>\theta / 3$ with $c \neq 0$, there exists a sequence $\left(d_{c, k}^{H}\right)$ such that, for any $p>0$ and $T$ large enough,

$$
\mathbf{P}\left(\hat{\theta}_{T} \geqslant c\right)=\frac{\exp (-T I(c)+P(c)) \sqrt{\sin (\pi H)}}{\sigma^{c} a^{c} \sqrt{2 \pi T}}\left[1+\sum_{k=1}^{p} \frac{d_{c, k}^{H}}{T^{k}}+\mathscr{O}\left(\frac{1}{T^{p+1}}\right)\right],
$$

where

$$
\begin{gathered}
P(c)=-\frac{1}{2} \ln \frac{(c-\theta)(3 c-\theta)}{4 c^{2}}, \\
a^{c}=2(c-\theta), \quad\left(\sigma^{c}\right)^{2}=\frac{c^{2}}{2(2 c-\theta)^{3}} .
\end{gathered}
$$

In addition, the coefficients $d_{c, 1}^{H}, \ldots, d_{c, p}^{H}$ can be calculated explicitly as in Theorem 2 .

c) Finally, for $c=0$, for any $p>0$ and for $T$ large enough,

$$
\mathbf{P}\left(\hat{\theta}_{T} \geqslant 0\right)=2 \frac{\exp (-T I(c)) \sqrt{\sin (\pi H)}}{\sqrt{2 \pi T} \sqrt{-2 \theta}}\left[1+\sum_{k=1}^{p} \frac{d_{k}^{H}}{T^{k}}+\mathscr{O}\left(\frac{1}{T^{p+1}}\right)\right] .
$$

As before, the coefficients $d_{1}^{H}, \ldots, d_{p}^{H}$ can be calculated explicitly. 
Theorem 6. For $c=\theta / 3$, there exists a sequence $\left(e_{k}^{H}\right)$ such that, for any $p>0$ and $T$ large enough,

$$
\mathbf{P}\left(\hat{\theta}_{T} \geqslant \frac{\theta}{3}\right)=\frac{\exp (-T I(c)) \Gamma(1 / 4)}{4 \pi T^{1 / 4} a_{\theta}^{3 / 4} \sigma_{\theta}} \sqrt{\sin (\pi H)}\left[1+\sum_{k=1}^{2 p} \frac{e_{k}^{H}}{(\sqrt{T})^{k}}+\mathscr{O}\left(\frac{1}{T^{p} \sqrt{T}}\right)\right],
$$

where $a_{\theta}$ and $\sigma_{\theta}$ are given by

$$
a_{\theta}=-\frac{4 \theta}{3}, \quad \sigma_{\theta}^{2}=-\frac{3}{2 \theta} .
$$

As before, the coefficients $e_{1}^{H}, \ldots, e_{2 p}^{H}$ can be calculated explicitly.

The proofs are left to the reader, since they follow essentially the same lines as that for the energy.

\section{Proofs of the main results.}

4.1. Proof of Theorem 2, first part. We first focus our attention on the SLDP for the energy $S_{T}$ in the easy case $-1 /(2 \theta)<c<-1 /\left(2 \theta \delta_{H}\right)$. Let $L_{T}$ be the normalized cumulant generating function of $S_{T}$. We already have seen that $a_{c}$, given by $(2.15)$, belongs to the domain $D_{H}$ whenever $c<-1 /\left(2 \theta \delta_{H}\right)$. For all $-1 /(2 \theta)<c<-1 /\left(2 \theta \delta_{H}\right)$, we can split $\mathbf{P}\left(S_{T} \geqslant c T\right)$ into two terms, $\mathbf{P}\left(S_{T} \geqslant c T\right)=A_{T} B_{T}$ with

$$
\begin{aligned}
& A_{T}=\exp \left(T\left(L_{T}\left(a_{c}\right)-c a_{c}\right)\right), \\
& B_{T}=\mathbf{E}_{T}\left[\exp \left(-a_{c}\left(S_{T}-c T\right)\right) \mathbf{1}_{S_{T} \geqslant c T}\right],
\end{aligned}
$$

where $\mathbf{E}_{T}$ stands for the expectation after the usual change of probability

$$
\frac{d \mathbf{P}_{T}}{d \mathbf{P}}=\exp \left(a_{c} S_{T}-T L_{T}\left(a_{c}\right)\right) .
$$

On the one hand, we can deduce from (1.9) with $L(a)=\mathscr{L}(0, a), H(a)=$ $\mathscr{H}(0, a), K_{T}(a)=\mathscr{K}_{T}(0, a)$, and $R_{T}(a)=\mathscr{R}_{T}(0, a)$ together with $(2.11)$ and (2.14) that

$$
\begin{aligned}
& A_{T}=\exp \left(T\left(L\left(a_{c}\right)-c a_{c}\right)+H\left(a_{c}\right)+K_{T}\left(a_{c}\right)+R_{T}\left(a_{c}\right)\right), \\
& A_{T}=\exp \left(-T I(c)+J(c)+K_{T}\left(a_{c}\right)+R_{T}\left(a_{c}\right)\right) .
\end{aligned}
$$

Consequently, we infer from (2.10) and (2.14) that for any $p>0$ and $T$ large enough

$$
K_{T}\left(a_{c}\right)=K_{H}(c)+\sum_{k=1}^{p} \frac{\gamma_{k}}{T^{k}}+\mathscr{O}\left(\frac{1}{T^{p+1}}\right),
$$

where the coefficients $\left(\gamma_{k}\right)$, which also depend on $H$, may be calculated explicitly. In addition, it is not hard to see from (1.13) that $R_{T}\left(a_{c}\right)=$ 
$\mathscr{O}(\exp (-T / c))$. Therefore, we deduce from (4.4) and (4.5) that for any $p>0$ and $T$ large enough

$$
A_{T}=\exp \left(-T I(c)+J(c)+K_{H}(c)\right)\left[1+\sum_{k=1}^{p} \frac{\alpha_{k}}{T^{k}}+\mathscr{O}\left(\frac{1}{T^{p+1}}\right)\right],
$$

where, as before, the coefficients $\left(\alpha_{k}\right)$ can be calculated explicitly. For example,

$$
\alpha_{1}=\frac{-2 c(1+2 \theta c) r_{1}^{H}}{\sin (\pi H)\left(2+(1+2 \theta c) p_{H}\right)} .
$$

It now remains to give the expansion for $B_{T}$ which can be rewritten as

$$
B_{T}=\mathbf{E}_{T}\left[\exp \left(-a_{c} \sigma_{c} \sqrt{T} U_{T}\right) \mathbf{1}_{U_{T} \geqslant 0}\right]
$$

where

$$
U_{T}=\frac{S_{T}-c T}{\sigma_{c} \sqrt{T}} .
$$

Lemma 2. For all $-1 /(2 \theta)<c<-1 /\left(2 \theta \delta_{H}\right)$, the distribution of $U_{T}$ under $\mathbf{P}_{T}$ converges, as $T$ goes to infinity, to the $\mathscr{N}(0,1)$ distribution. Moreover, there exists a sequence $\left(\beta_{k}\right)$ such that, for any $p>0$ and $T$ large enough,

$$
B_{T}=\frac{\beta_{0}}{\sqrt{T}}\left[1+\sum_{k=1}^{p} \frac{\beta_{k}}{T^{k}}+\mathscr{O}\left(\frac{1}{T^{p+1}}\right)\right] .
$$

The sequence $\left(\beta_{k}\right)$ only depends on the derivatives of $L$ and $H$ evaluated at point $a_{c}$. They also depend on the Taylor expansion of $K_{T}$ and its derivatives at $a_{c}$. In particular,

$$
\begin{aligned}
& \beta_{0}=\frac{1}{a_{c} \sigma_{c} \sqrt{2 \pi}}, \\
& \beta_{1}=\frac{1}{\sigma_{c}^{2}}\left(\frac{s_{1}}{a_{c}}-\frac{s_{1}^{2}}{2}-\frac{s_{2}}{2}-\frac{s_{3}}{2 a_{c} \sigma_{c}^{2}}+\frac{s_{1} \ell_{3}}{2 \sigma_{c}^{2}}-\frac{5 \ell_{3}^{2}}{24 \sigma_{c}^{4}}+\frac{\ell_{4}}{8 \sigma_{c}^{2}}-\frac{1}{a_{c}^{2}}\right),
\end{aligned}
$$

where $\ell_{q}=L^{(q)}\left(a_{c}\right), h_{q}=H^{(q)}\left(a_{c}\right)$, and $s_{q}=h_{q}+k_{q}$.

The proof of Lemma 2 is given in Section 6.1.

Pro of of The or e m 2, first part. The expansions (2.12) and (2.13) immediately follow from the conjunction of (4.6) and (4.8).

4.2. Proof of Theorem 2, second part. We are now going to establish the SLDP for the energy $S_{T}$ in the more complicated case $c>-1 /\left(2 \theta \delta_{H}\right)$. We have already seen that the point $a_{c}$, given by (2.11), belongs to the domain $\left.D_{H}=\right]-\infty, a_{H}$ [ whenever $c<-1 /\left(2 \theta \delta_{H}\right)$. Consequently, for $c>-1 /\left(2 \theta \delta_{H}\right)$, it is necessary to make use of a slight modification of the strategy of time varying change of probability proposed in [6].

Now, we show a modification of the decomposition (1.9) which allows us to replace $r_{T}(a)$ by $p_{H}$ in $K_{T}$, putting the difference into the remainder term. The modified remainder will be denoted by $\check{R}_{T}$. 
Lemma 3. For any $a \in D_{H}$, we have the following decomposition:

$$
L_{T}(a)=L(a)+\frac{1}{T} H(a)+\frac{1}{T} K(a)+\frac{1}{T} \check{R}_{T}(a),
$$

where $L$ and $H$ are given by (2.8) and (2.9), respectively,

$$
K(a)=-\frac{1}{2} \ln \left(1+\frac{\varphi(a)+\theta}{2 \varphi(a)} p_{H}\right),
$$

and the remainder term has the form

$$
\begin{aligned}
\check{R}_{T}(a)=-\frac{1}{2} \ln (1 & +\frac{(\varphi(a)+\theta)\left(r_{T}(a)-p_{H}\right)}{\left(2+p_{H}\right) \varphi(a)+\theta \delta_{H}} \\
& \left.+\frac{(\varphi(a)+\theta)^{2}}{(\varphi(a)-\theta)\left(\left(2+p_{H}\right) \varphi(a)+\theta \delta_{H}\right)} e^{-2 T \varphi(a)}\right) .
\end{aligned}
$$

P r o o f. It follows from (1.9) that

$$
\begin{aligned}
L_{T}(a) & =L(a)+\frac{1}{T} H(a)+\frac{1}{T} K_{T}(a)+\frac{1}{T} R_{T}(a) \\
& =L(a)+\frac{1}{T} H(a)+\frac{1}{T} K(a)+\frac{1}{T} \check{R}_{T}(a),
\end{aligned}
$$

where

$$
\check{R}_{T}(a)=K_{T}(a)-K(a)+R_{T}(a) .
$$

Hence, if we denote $\varphi=\sqrt{\theta^{2}-2 a}$, then it is not hard to see that

$$
\begin{aligned}
\exp \left(-2 \check{R}_{T}(a)\right)= & \frac{2 \varphi+(\varphi+\theta) r_{T}(a)}{2 \varphi+(\varphi+\theta) p_{H}} \\
& \times\left(1+\frac{(\varphi+\theta)^{2}}{(\varphi-\theta)\left(2 \varphi+(\varphi+\theta) r_{T}(a)\right)} e^{-2 T \varphi}\right) \\
= & \frac{2 \varphi+(\varphi+\theta) r_{T}(a)}{2 \varphi+(\varphi+\theta) p_{H}}+\frac{(\varphi+\theta)^{2}}{(\varphi-\theta)\left(2 \varphi+(\varphi+\theta) p_{H}\right)} e^{-2 T \varphi} \\
= & 1+\frac{(\varphi+\theta)\left(r_{T}(a)-p_{H}\right)}{2 \varphi+(\varphi+\theta) p_{H}}+\frac{(\varphi+\theta)^{2}}{(\varphi-\theta)\left(2 \varphi+(\varphi+\theta) p_{H}\right)} e^{-2 T \varphi}
\end{aligned}
$$

which ends the proof of Lemma 3.

Denote by $\Lambda_{T}$ the suitable approximation of the normalized cumulant generating function $L_{T}$ given by

$$
\Lambda_{T}(a)=L(a)+\frac{1}{T} H(a)+\frac{1}{T} K(a) .
$$

The previous lemma enable us to write

$$
L_{T}(a)=\Lambda_{T}(a)+\frac{1}{T} \check{R}_{T}(a) .
$$


One can observe that $\Lambda_{T}$ is a holomorphic function in the domain $D_{H}$. In addition, there exists a unique $a_{T}$, which belongs to the interior of $D_{H}$ and converges to its border $a_{H}$, solution of the implicit equation

$$
\Lambda_{T}^{\prime}(a)=c .
$$

After some tedious but straightforward calculations, we can deduce from (4.14) that there exists a sequence $\left(a_{k}\right)$ such that, for any $p>0$ and $T$ large enough,

$$
a_{T}=\sum_{k=0}^{p} \frac{a_{k}}{T^{k}}+\mathscr{O}\left(\frac{1}{T^{p+1}}\right)
$$

with

$$
\begin{gathered}
a_{0}=a_{H}, \quad a_{1}=-\frac{\theta \delta_{H}}{1+2 \theta c \delta_{H}}, \\
a_{2}=\frac{2 \theta c \delta_{H}(4+\sin (\pi H))+2+\sin (\pi H)}{2\left(1+2 \theta c \delta_{H}\right)^{3}} .
\end{gathered}
$$

Moreover, if $\varphi_{T}=\varphi\left(a_{T}\right)=\sqrt{\theta^{2}-2 a_{T}}$, then we also have for any $p>0$ and $T$ large enough,

$$
\varphi_{T}=\sum_{k=0}^{p} \frac{\varphi_{k}}{T^{k}}+\mathscr{O}\left(\frac{1}{T^{p+1}}\right)
$$

with

$$
\begin{gathered}
\varphi_{0}=-\theta \delta_{H}, \quad \varphi_{1}=\frac{-1}{1+2 \theta c \delta_{H}} \\
\varphi_{2}=\frac{2 \theta c \delta_{H}(5+\sin (\pi H))+3+\sin (\pi H)}{2 \theta \delta_{H}\left(1+2 \theta c \delta_{H}\right)^{3}}
\end{gathered}
$$

Hereafter, we introduce the new probability measure

$$
\frac{d \mathbf{P}_{T}}{d \mathbf{P}}=\exp \left(a_{T} S_{T}-T L_{T}\left(a_{T}\right)\right)
$$

and we denote by $\mathbf{E}_{T}$ the expectation under $\mathbf{P}_{T}$. It clearly leads to the decomposition $\mathbf{P}\left(S_{T} \geqslant c T\right)=A_{T} B_{T}$, where

$$
\begin{aligned}
& A_{T}=\exp \left(T L_{T}\left(a_{T}\right)-c T a_{T}\right) \\
& B_{T}=\mathbf{E}_{T}\left[\exp \left(-a_{T}\left(S_{T}-c T\right)\right) \mathbf{1}_{S_{T} \geqslant c T}\right] .
\end{aligned}
$$

The proof now splits into two parts, the first one is devoted to the expansion of $A_{T}$ while the second one gives the expansion of $B_{T}$. It follows from (4.12), (4.13) and (4.18) that

$$
A_{T}=\exp \left(T\left(L\left(a_{T}\right)-c a_{T}\right)+H\left(a_{T}\right)+K\left(a_{T}\right)+\check{R}_{T}\left(a_{T}\right)\right) .
$$


We can deduce from the Taylor expansions of $a_{T}$ and $\varphi_{T}$ given by (4.15) and (4.16) that

$$
\begin{aligned}
& T\left(L\left(a_{T}\right)-c a_{T}\right)=-\frac{T}{2}\left(\theta+\varphi_{T}+2 c a_{T}\right) \\
& \quad=-T\left(c a_{H}-L\left(a_{H}\right)\right)-\frac{\varphi_{1}}{2}-c a_{1}-\frac{1}{2} \sum_{k=1}^{p} \frac{\varphi_{k+1}+2 c a_{k+1}}{T^{k}}+\mathscr{O}\left(\frac{1}{T^{p+1}}\right) \\
& \quad=-T I(c)+\frac{1}{2}-\frac{1}{2} \sum_{k=1}^{p} \frac{\varphi_{k+1}+2 c a_{k+1}}{T^{k}}+\mathscr{O}\left(\frac{1}{T^{p+1}}\right) .
\end{aligned}
$$

Consequently, we obtain that for any $p>0$ and $T$ large enough,

$$
\exp \left(T\left(L\left(a_{T}\right)-c a_{T}\right)\right)=\exp (-T I(c)) \sqrt{e}\left[1+\sum_{k=1}^{p} \frac{\alpha_{k}}{T^{k}}+\mathscr{O}\left(\frac{1}{T^{p+1}}\right)\right]
$$

where the coefficients $\left(\alpha_{k}\right)$ can be calculated explicitly. For example,

$$
\alpha_{1}=\frac{-1}{4 \theta \delta_{H}\left(1+2 c \theta \delta_{H}\right)^{2}}\left(2 \theta c \delta_{H}(4+\sin (\pi H))+3+\sin (\pi H)\right) .
$$

By the same way, we find that for any $p>0$ and $T$ large enough,

$$
\exp \left(H\left(a_{T}\right)\right)=\sqrt{\frac{2 \varphi_{T}}{\varphi_{T}-\theta}}=\sqrt{1-\sin (\pi H)}\left[1+\sum_{k=1}^{p} \frac{\beta_{k}}{T^{k}}+\mathscr{O}\left(\frac{1}{T^{p+1}}\right)\right],
$$

where the coefficients $\left(\beta_{k}\right)$ can be calculated explicitly. For example,

$$
\beta_{1}=\frac{1+\sin (\pi H)}{4 \theta \delta_{H}\left(1+2 \theta c \delta_{H}\right)} .
$$

The expansions for $K\left(a_{T}\right)$ and $\check{R}_{T}\left(a_{T}\right)$ are much more tricky. On the one hand,

$$
\exp \left(K\left(a_{T}\right)\right)=\sqrt{\frac{2 \varphi_{T}}{2 \varphi_{T}+\left(\varphi_{T}+\theta\right) p_{H}}} .
$$

One can observe that $2 \varphi_{0}+\left(\varphi_{0}+\theta\right) p_{H}=0$. Hence, multiplying the numerator and the denominator by $T$, we obtain that for any $p>0$ and $T$ large enough,

$$
\begin{aligned}
\exp \left(K\left(a_{T}\right)\right)= & \sqrt{\frac{2 T \varphi_{T}}{2 T \varphi_{T}+T\left(\varphi_{T}+\theta\right) p_{H}}}=\sqrt{\theta T \delta_{H}\left(1-\delta_{H}\right)\left(1+2 \theta c \delta_{H}\right)} \\
& \times\left[1+\sum_{k=1}^{p} \frac{\gamma_{k}}{T^{k}}+\mathscr{O}\left(\frac{1}{T^{p+1}}\right)\right],
\end{aligned}
$$


where, as before, the coefficients $\left(\gamma_{k}\right)$ can be calculated explicitly. On the other hand, $\check{R}_{T}\left(a_{T}\right)=K_{T}\left(a_{T}\right)-K\left(a_{T}\right)+R_{T}\left(a_{T}\right)$. It is not hard to see that

$$
\begin{aligned}
\exp \left(-2 \check{R}_{T}\left(a_{T}\right)\right)= & \frac{2 \varphi_{T}+\left(\varphi_{T}+\theta\right) r_{T}\left(a_{T}\right)}{2 \varphi_{T}+\left(\varphi_{T}+\theta\right) p_{H}} \\
& +\frac{\left(\varphi_{T}+\theta\right)^{2}}{\left(\varphi_{T}-\theta\right)\left(2 \varphi_{T}+\left(\varphi_{T}+\theta\right) p_{H}\right)} \exp \left(-2 T \varphi_{T}\right), \\
= & \frac{2 \varphi_{T}+\left(\varphi_{T}+\theta\right) r_{T}\left(a_{T}\right)}{2 \varphi_{T}+\left(\varphi_{T}+\theta\right) p_{H}}+\mathscr{O}\left(T \exp \left(2 \theta T \delta_{H}\right)\right) .
\end{aligned}
$$

Therefore,

$$
\exp \left(\check{R}_{T}\left(a_{T}\right)\right)=\sqrt{\frac{2 \varphi_{T}+\left(\varphi_{T}+\theta\right) p_{H}}{2 \varphi_{T}+\left(\varphi_{T}+\theta\right) r_{T}\left(a_{T}\right)}}\left(1+\mathscr{O}\left(T \exp \left(2 \theta T \delta_{H}\right)\right)\right) .
$$

Recall that $r_{T}(a)=r_{H}(\varphi(a) T / 2) \exp (-T \varphi(a))-1$. It is shown (equation (5.9) in Section 5) that for any $p>0$ and $T$ large enough,

$$
r_{T}\left(a_{T}\right)=p_{H}+\frac{1}{\sin (\pi H)} \sum_{k=1}^{p} \frac{2^{k} r_{k}^{H}}{\varphi_{T}^{k} T^{k}}+\mathscr{O}\left(\frac{1}{T^{p+1}}\right)
$$

where the coefficients $\left(r_{k}^{H}\right)$ can be calculated explicitly. For example,

$$
r_{1}^{H}=-\frac{(2 H-1)^{2}}{4}
$$

Consequently, we infer from (4.24) that

$$
T\left(r_{T}\left(a_{T}\right)-p_{H}\right)=w_{T}\left(a_{T}\right)+\mathscr{O}\left(\frac{1}{T^{p}}\right),
$$

where

$$
w_{T}\left(a_{T}\right)=\frac{1}{\sin (\pi H)} \sum_{k=1}^{p} \frac{2^{k} r_{k}^{H}}{\varphi_{T}^{k} T^{k-1}} .
$$

If $\mu_{T}=T\left(2 \varphi_{T}+\left(\varphi_{T}+\theta\right) p_{H}\right)$, then we obtain that for any $p>0$ and $T$ large enough,

$$
\begin{aligned}
\exp \left(\check{R}_{T}\left(a_{T}\right)\right)= & \sqrt{\frac{\mu_{T}}{\mu_{T}+\left(\varphi_{T}+\theta\right) T\left(r_{T}\left(a_{T}\right)-p_{H}\right)}}\left(1+\mathscr{O}\left(T \exp \left(2 \theta T \delta_{H}\right)\right)\right) \\
= & \sqrt{\frac{1-\sin ^{2}(\pi H)}{1-\sin ^{2}(\pi H)+4 r_{1}^{H} \sin (\pi H)\left(1+2 \theta c \delta_{H}\right)}} \\
& \times\left[1+\sum_{k=1}^{p} \frac{\delta_{k}}{T^{k}}+\mathscr{O}\left(\frac{1}{T^{p+1}}\right)\right]
\end{aligned}
$$


where, as before, the coefficients $\left(\delta_{k}\right)$ can be calculated explicitly. Putting together the four contributions (4.21), (4.22), (4.23), and (4.25), we find from (4.20) that for any $p>0$ and $T$ large enough,

$$
\begin{aligned}
A_{T}= & \exp \left(-T I(c)+R_{H}(c)\right) \delta_{H} \sqrt{2 e \theta T \sin (\pi H)\left(1+2 \theta c \delta_{H}\right)} \\
& \times\left[1+\sum_{k=1}^{p} \frac{\alpha_{k}}{T^{k}}+\mathscr{O}\left(\frac{1}{T^{p+1}}\right)\right]
\end{aligned}
$$

where the coefficients $\left(\alpha_{k}\right)$ can be calculated explicitly and

$$
R_{H}(c)=-\frac{1}{2} \ln \left(1-\frac{(2 H-1)^{2} \sin (\pi H)\left(1+2 \theta c \delta_{H}\right)}{1-\sin ^{2}(\pi H)}\right) .
$$

The rest of the proof concerns the expansion of $B_{T}$ which can be rewritten as

$$
B_{T}=\mathbf{E}_{T}\left[\exp \left(-a_{T} T U_{T}\right) \mathbf{1}_{U_{T} \geqslant 0}\right]
$$

where

$$
U_{T}=\frac{S_{T}-c T}{T} .
$$

Lemma 4. For all $c>-1 /\left(2 \theta \delta_{H}\right)$, the distribution of $U_{T}$ under $\mathbf{P}_{T}$ converges, as $T$ goes to infinity, to the distribution of $\nu_{H} N^{2}-\gamma_{H}$, where $N$ stands for the standard $\mathscr{N}(0,1)$ distribution,

$$
\begin{gathered}
\gamma_{H}=c-L^{\prime}\left(a_{H}\right)=\frac{1+2 \theta c \delta_{H}}{2 \theta \delta_{H}}, \\
\nu_{H}=\frac{\left(1-\sin ^{2}(\pi H)\right) \gamma_{H}}{1-\sin ^{2}(\pi H)-(2 H-1)^{2} \sin (\pi H)\left(1+2 \theta c \delta_{H}\right)} .
\end{gathered}
$$

In other words, the limit of the characteristic function of $U_{T}$ under $\mathbf{E}_{T}$ is

$$
\Phi(u)=\frac{\exp \left(-i \gamma_{H} u\right)}{\sqrt{1-2 i \nu_{H} u}} .
$$

Moreover, there exists a sequence $\left(\beta_{k}\right)$ such that, for any $p>0$ and $T$ large enough,

$$
B_{T}=\sum_{k=1}^{p} \frac{\beta_{k}}{T^{k}}+\mathscr{O}\left(\frac{1}{T^{p+1}}\right) .
$$

The coefficients $\left(\beta_{k}\right)$ only depend on the Taylor expansion of $a_{T}$ at the neighborhood of $a_{H}$ together with the derivatives of $L$ and $H$ evaluated at point $a_{H}$. They also depend on the Taylor expansion of $K_{T}$ and its derivatives at $a_{H}$. In particular,

$$
\beta_{1}=\frac{1}{a_{H} \gamma_{H} \sqrt{2 \pi e}} \exp \left(Q_{H}(c)-R_{H}(c)\right)
$$


where $R_{H}(c)$ is given by (4.27) and

$$
Q_{H}(c)=\frac{(2 H-1)^{2} \sin (\pi H)\left(1+2 \theta c \delta_{H}\right)}{2\left(1-\sin ^{2}(\pi H)\right)} .
$$

The proof of Lemma 4 is given in Section 6.2.

P r o of of $\mathrm{T} \mathrm{h}$ e or e m 2, s e c o n d part. The expansions (4.26) and (4.32) imply (2.16), which completes the proof of Theorem 2.

4.3. Proof of Theorem 3. We shall now proceed to the proof of Theorem 3 which essentially follows the same lines as that of Theorem 2, second part. First of all, one can observe that if $c=-1 /\left(2 \theta \delta_{H}\right)$, then we exactly have $a_{c}=a_{H}$. As in the proof of Theorem 2, there exists a unique $a_{T}$, which belongs to the interior of $\left.D_{H}=\right]-\infty, a_{H}$ [ and converges to its border $a_{H}$, solution of the implicit equation

$$
\Lambda_{T}^{\prime}(a)=c=-\frac{1}{2 \theta \delta_{H}},
$$

where $\Lambda_{T}$ is given by (4.12). We deduce from (4.33) that

$$
T\left(\varphi_{T}+\theta \delta_{H}\right)^{2}=\frac{\theta\left(\varphi_{T}+\theta p_{H}\right)}{c \varphi_{T}\left(\varphi_{T}-\theta\right)\left(2+p_{H}\right)} .
$$

Consequently, we infer from (4.33) and (4.34) that there exists a sequence $\left(a_{k}\right)$ such that, for any $p>0$ and $T$ large enough,

$$
a_{T}=\sum_{k=0}^{2 p} \frac{a_{k}}{(\sqrt{T})^{k}}+\mathscr{O}\left(\frac{1}{T^{p} \sqrt{T}}\right), \quad \varphi_{T}=\sum_{k=0}^{2 p} \frac{\varphi_{k}}{(\sqrt{T})^{k}}+\mathscr{O}\left(\frac{1}{T^{p} \sqrt{T}}\right)
$$

with

$$
\begin{gathered}
a_{0}=a_{H}, \quad \varphi_{0}=-\theta \delta_{H}, \quad a_{1}=-\left(-\theta \delta_{H}\right)^{3 / 2}, \quad \varphi_{1}=\sqrt{-\theta \delta_{H}}, \\
a_{2}=-\frac{\theta \delta_{H}}{4}(1+\sin (\pi H)), \quad \varphi_{2}=-\frac{1}{4}(3+\sin (\pi H)) .
\end{gathered}
$$

Furthermore, we have the decomposition $\mathbf{P}\left(S_{T} \geqslant c T\right)=A_{T} B_{T}$, where $A_{T}$ and $B_{T}$ are given by (4.18) and (4.19), respectively. Via the same lines as in the proof of the expansion (4.26), we find that for any $p>0$ and $T$ large enough,

$$
\begin{aligned}
A_{T}= & \exp (-T I(c))\left(-\theta \delta_{H} e T\right)^{1 / 4} \sqrt{2 \delta_{H} \sin (\pi H)} \\
& \times\left[1+\sum_{k=1}^{2 p} \frac{\alpha_{k}}{(\sqrt{T})^{k}}+\mathscr{O}\left(\frac{1}{T^{p} \sqrt{T}}\right)\right]
\end{aligned}
$$


where the coefficients $\left(\alpha_{k}\right)$ can be calculated explicitly. It still remains to give the expansion of $B_{T}$ which can be rewritten as

$$
B_{T}=\mathbf{E}_{T}\left[\exp \left(-a_{T} \sqrt{T} U_{T}\right) \mathbf{1}_{U_{T} \geqslant 0}\right],
$$

where

$$
U_{T}=\frac{S_{T}-c T}{\sqrt{T}} .
$$

Lemma 5. For $c=-1 /\left(2 \theta \delta_{H}\right)$, the distribution of $U_{T}$ under $\mathbf{P}_{T}$ converges, as $T$ goes to infinity, to the distribution of $\sigma_{H} N_{1}+\eta_{H}\left(N_{2}^{2}-1\right)$, where $N_{1}$ and $N_{2}$ are two independent $\mathscr{N}(0,1)$ random variables and

$$
\begin{aligned}
\sigma_{H}^{2} & =L^{\prime \prime}\left(a_{H}\right)=-\frac{1}{2\left(\theta \delta_{H}\right)^{3}}, \\
\eta_{H} & =\frac{1}{2\left(-\theta \delta_{H}\right)^{3 / 2}} .
\end{aligned}
$$

In other words, the limit of the characteristic function of $U_{T}$ under $\mathbf{E}_{T}$ is

$$
\Phi(u)=\frac{\exp \left(-i \eta_{H} u-u^{2} \sigma_{H}^{2} / 2\right)}{\sqrt{1-2 i \eta_{H} u}} .
$$

Moreover, there exists a sequence $\left(\beta_{k}\right)$ such that, for any $p>0$ and $T$ large enough,

$$
B_{T}=\sum_{k=1}^{2 p} \frac{\beta_{k}}{(\sqrt{T})^{k}}+\mathscr{O}\left(\frac{1}{T^{p} \sqrt{T}}\right)
$$

where the sequence $\left(\beta_{k}\right)$ can be calculated explicitly. In particular,

$$
\beta_{1}=\frac{1}{4 \pi a_{H} \eta_{H}} \exp \left(-\frac{1}{4}\right) \Gamma\left(\frac{1}{4}\right) .
$$

The proof of Lemma 5 is given in Section 6.3.

P r o of of $\mathrm{T} \mathrm{h}$ e or e m 3. The expansions (4.36) and (4.42) imply (2.19), which completes the proof of Theorem 3 .

5. Appendix A: On the main asymptotic expansion. We shall first prove the asymptotic expansion (1.9) of the normalized cumulant generating function $\mathscr{L}_{T}(a, b)$. This result was partially established by formula (5.12) in [12]. By Girsanov's theorem, $\mathscr{L}_{T}(a, b)$ can be rewritten as

$$
\begin{aligned}
\mathscr{L}_{T}(a, b) & =\frac{1}{T} \ln \mathbf{E}\left[\exp \left(a \int_{0}^{T} Q_{t} d Y_{t}+b S_{T}\right)\right] \\
& =\frac{1}{T} \ln \mathbf{E}_{\varphi}\left[\exp \left((a+\theta-\varphi) \int_{0}^{T} Q_{t} d Y_{t}+\frac{1}{2}\left(2 b-\theta^{2}+\varphi^{2}\right) S_{T}\right)\right]
\end{aligned}
$$


for all $\varphi \in \mathbf{R}$, where $\mathbf{E}_{\varphi}$ stands for the expectation after the usual change of probability

$$
\frac{d \mathbf{P}_{\varphi}}{d \mathbf{P}}=\exp \left((\varphi-\theta) \int_{0}^{T} Q_{t} d Y_{t}-\frac{1}{2}\left(\varphi^{2}-\theta^{2}\right) S_{T}\right)
$$

If $\theta^{2}-2 b>0$, we can choose $\varphi=\sqrt{\theta^{2}-2 b}$ and $\tau=\varphi-(a+\theta)$ which leads to

$$
\mathscr{L}_{T}(a, b)=\frac{1}{T} \ln \mathbf{E}_{\varphi}\left[\exp \left(-\tau \int_{0}^{T} Q_{t} d Y_{t}\right)\right]
$$

By Itô's formula, we also have

$$
\int_{0}^{T} Q_{t} d Y_{t}=\frac{1}{2}\left(l_{H} Y_{T} \int_{0}^{T} t^{2 H-1} d Y_{t}-T\right)
$$

Consequently, we obtain from (5.1) that

$$
\mathscr{L}_{T}(a, b)=\frac{\tau}{2}+\frac{1}{T} \ln \mathbf{E}_{\varphi}\left[\exp \left(-\frac{\tau l_{H}}{2} Y_{T} \int_{0}^{T} t^{2 H-1} d Y_{t}\right)\right] .
$$

Under the new probability $\mathbf{P}_{\varphi}$, the pair $\left(Y_{T}, \int_{0}^{T} t^{2 H-1} d Y_{t}\right)$ is Gaussian with mean zero and covariance matrix $\Gamma_{T}(\varphi)$. Denote by $I$ and $J$ the two matrices

$$
I=\left(\begin{array}{ll}
1 & 0 \\
0 & 1
\end{array}\right) \quad \text { and } \quad J=\left(\begin{array}{ll}
0 & 1 \\
1 & 0
\end{array}\right)
$$

As soon as the matrix

$$
M_{T}(a, b)=I+\frac{\tau l_{H}}{2} \Gamma_{T}^{1 / 2}(\varphi) J \Gamma_{T}^{1 / 2}(\varphi)
$$

is positive definite, we deduce from (5.2) together with standard calculus on the Gaussian distribution that

$$
\mathscr{L}_{T}(a, b)=\frac{\tau}{2}-\frac{1}{2 T} \ln \operatorname{det}\left(M_{T}(a, b)\right) .
$$

Furthermore, it have been already proven by relation (5.12) of [12] that, if $\tau>0$, then

$$
\operatorname{det}\left(M_{T}(a, b)\right)=\frac{1}{z_{T}}\left[x_{T}\left(1+\frac{\tau}{\varphi} e^{\delta_{T}} \operatorname{sh} \delta_{T}\right)^{2}-y_{T}\left(1-\frac{\tau}{\varphi} e^{\delta_{T}} \operatorname{ch} \delta_{T}\right)^{2}\right]
$$

with $\delta_{T}=T \varphi / 2, x_{T}=I_{H-1}\left(\delta_{T}\right) I_{-H}\left(\delta_{T}\right), y_{T}=I_{1-H}\left(\delta_{T}\right) I_{H}\left(\delta_{T}\right)$, and

$$
z_{T}=x_{T}-y_{T}=\frac{4 \sin (\pi H)}{\pi \varphi T},
$$


where $I_{H}$ is the modified Bessel function of the first kind. We refer the reader to [13, Chap. 5] for the main properties of Bessel functions. Therefore, if $p_{T}=\left(x_{T}+y_{T}\right) / z_{T}$ and $r_{T}=2 p_{T} e^{-T \varphi}-1$, then we deduce from (5.4) after some straightforward calculations that

$$
\begin{aligned}
\operatorname{det}\left(M_{T}(a, b)\right) & =\frac{(2 \varphi-\tau)^{2}}{4 \varphi^{2}}+p_{T} \frac{\tau(2 \varphi-\tau)}{2 \varphi^{2}} e^{T \varphi}+\frac{\tau^{2}}{4 \varphi^{2}} e^{2 T \varphi} \\
& =\frac{\tau}{2 \varphi} e^{2 T \varphi}\left(1+\frac{2 \varphi-\tau}{2 \varphi} r_{T}+\frac{(2 \varphi-\tau)^{2}}{2 \varphi \tau} e^{-2 T \varphi}\right) .
\end{aligned}
$$

Consequently, we infer from (5.3) and (5.5) that

$$
\begin{aligned}
\mathscr{L}_{T}(a, b)= & -\frac{1}{2}(a+\theta+\varphi)-\frac{1}{2 T} \ln \frac{\tau}{2 \varphi}-\frac{1}{2 T} \ln \left(1+\frac{2 \varphi-\tau}{2 \varphi} r_{T}\right) \\
& -\frac{1}{2 T} \ln \left(1+\frac{(2 \varphi-\tau)^{2}}{\tau\left(2 \varphi+r_{T}(2 \varphi-\tau)\right)} e^{-2 T \varphi}\right) .
\end{aligned}
$$

In order to complete the proof of Lemma 1, it remains to show that the limiting domain $\Delta_{H}$ reduces to $\theta^{2}-2 b>0$ and $\sqrt{\theta^{2}-2 b}>\max \left(a+\theta ;-\delta_{H}(a+\theta)\right)$. On the one hand, we have already seen that our calculation is true as soon as $\theta^{2}-2 b>0$ and $\tau>0$ which can be rewritten as

$$
\varphi>a+\theta \text {. }
$$

On the other hand, we also have the second constraint

$$
1+\frac{2 \varphi-\tau}{2 \varphi} r_{T}>0
$$

leading to

$$
\sqrt{\theta^{2}-2 b}>-\delta_{H}(a+\theta) \text {. }
$$

As a matter of fact, it follows from the asymptotic expansion (5.11.10) of [13] for the Bessel function $I_{H}$ that for all $z \in \mathbf{C}$ with $|z|$ large enough and $|\arg (z)| \leqslant \pi / 2-\delta$, where $\delta$ is an arbitrarily small positive number, and for any $p>0$

$$
r_{H}(z)=\frac{\exp (2 z)}{\sin (\pi H)}\left[1+\sum_{k=1}^{p} \frac{r_{k}^{H}}{z^{k}}+\mathscr{O}\left(\frac{1}{|z|^{p+1}}\right)\right] .
$$

Moreover, the coefficients $\left(r_{k}^{H}\right)$ can be calculated explicitly. For example, one can check that $r_{1}^{H}=-(2 H-1)^{2} / 4$ and $r_{2}^{H}=(2 H-1)^{2}(2 H+1)(2 H-3) / 32$. In addition, all the coefficients $\left(r_{k}^{H}\right)$ vanish if $H=1 / 2$. Consequently,

$$
r_{T}(a)=p_{H}+\frac{1}{\sin (\pi H)} \sum_{k=1}^{p} \frac{2^{k} r_{k}^{H}}{(\varphi(a))^{k} T^{k}}+\mathscr{O}\left(\frac{1}{T^{p+1}}\right)
$$

with $p_{H}=(1-\sin (\pi H)) / \sin (\pi H)$. Hence, as $T$ tends to infinity, (5.6) reduces to $2 \varphi+(\varphi+(a+\theta)) p_{H}>0$ so $\varphi\left(2+p_{H}\right)>-p_{H}(a+\theta)$. Finally, since $\delta_{H}=p_{H} /\left(2+p_{H}\right)$, it clearly implies (5.7) which completes the proof of Lemma 1. 


\section{Appendix B: On the characteristic functions.}

6.1. Proof of Lemma 2. If $\Phi_{T}$ denotes the characteristic function of $U_{T}$ under $\mathbf{P}_{T}$, then it follows from (4.3) that

$$
\Phi_{T}(u)=\exp \left(-\frac{i u c \sqrt{T}}{\sigma_{c}}+T\left(L_{T}\left(a_{c}+\frac{i u}{\sigma_{c} \sqrt{T}}\right)-L_{T}\left(a_{c}\right)\right)\right) .
$$

First of all, it is necessary to prove that for $T$ large enough, $\Phi_{T}$ belongs to $L^{2}(\mathbf{R})$. One can observe that, in contrast with [3], it is impossible here to make use of the Karhunen-Loève expansion of the process $\left(X_{t}\right)$.

Lemma 6. For $T$ large enough, $\Phi_{T}$ belongs to $L^{2}(\mathbf{R})$.

$\mathrm{P}$ r o o f. It is a direct consequence of Proposition 4 in Section 7.1. We shall now establish an asymptotic expansion for the characteristic function $\Phi_{T}$, similar to that of Lemma 7.1 of [3].

Lemma 7. For any $p>0$, there exist integers $q(p), r(p)$, and a sequence $\left(\varphi_{k, l}^{H}\right)$ independent of $p$ such that for $T$ large enough

$$
\Phi_{T}(u)=\exp \left(-\frac{u^{2}}{2}\right)\left[1+\frac{1}{\sqrt{T}} \sum_{k=0}^{2 p} \sum_{l=k+1}^{q(p)} \frac{\varphi_{k, l}^{H} u^{l}}{(\sqrt{T})^{k}}+\mathscr{O}\left(\frac{\max \left\{1,|u|^{r(p)}\right\}}{T^{p+1}}\right)\right]
$$

and the remainder $\mathscr{O}$ is uniform as soon as $|u| \leqslant s T^{1 / 6}$ for some positive constant $s$.

P r o o f. It is rather easy to see that for all $k \in \mathbf{N}, R_{T}^{(k)}\left(a_{c}\right)=$ $\mathscr{O}\left(T^{k} \exp (-T / c)\right)$. Hence, we infer from (1.9) together with $(2.8)-(2.10)$ that for all $k \in \mathbf{N}$,

$$
L_{T}^{(k)}\left(a_{c}\right)=L^{(k)}\left(a_{c}\right)+\frac{1}{T} H^{(k)}\left(a_{c}\right)+\frac{1}{T} K_{T}^{(k)}\left(a_{c}\right)+\mathscr{O}\left(T^{k} \exp \left(-\frac{T}{c}\right)\right) .
$$

Therefore, we find from (6.1) and (6.3) that for any $p>0$,

$$
\begin{aligned}
\ln \Phi_{T}(u)= & -\frac{u^{2}}{2}+T \sum_{k=3}^{2 p+3}\left(\frac{i u}{\sigma_{c} \sqrt{T}}\right)^{k} \frac{L^{(k)}\left(a_{c}\right)}{k !} \\
& +\sum_{k=1}^{2 p+1}\left(\frac{i u}{\sigma_{c} \sqrt{T}}\right)^{k} \frac{H^{(k)}\left(a_{c}\right)+K_{T}^{(k)}\left(a_{c}\right)}{k !}+\mathscr{O}\left(\frac{\max \left\{1, u^{2 p+4}\right\}}{T^{p+1}}\right) .
\end{aligned}
$$

We deduce the asymptotic expansion (6.2) by taking the exponential on both sides, remarking that, as soon as $|u| \leqslant s T^{1 / 6}$ for some positive constant $s$, the quantity $u^{l} /(\sqrt{T})^{k}$ in (6.2) remains bounded.

$\mathrm{P}$ r o of of $\mathrm{L} \mathrm{e} \mathrm{m} \mathrm{m} \mathrm{a} \mathrm{2.} \mathrm{It} \mathrm{follows} \mathrm{from} \mathrm{Parseval's} \mathrm{formula} \mathrm{that} B_{T}$, given by (4.7), can be rewritten as

$$
B_{T}=\frac{1}{2 \pi a_{c} \sigma_{c} \sqrt{T}} \int_{\mathbf{R}}\left(1+\frac{i u}{a_{c} \sigma_{c} \sqrt{T}}\right)^{-1} \Phi_{T}(u) d u .
$$


For some positive constant $s$, set $s_{T}=s T^{1 / 6}$. We can split $B_{T}=C_{T}+D_{T}$, where

$$
\begin{aligned}
C_{T} & =\frac{1}{2 \pi a_{c} \sigma_{c} \sqrt{T}} \int_{|u| \leqslant s_{T}}\left(1+\frac{i u}{a_{c} \sigma_{c} \sqrt{T}}\right)^{-1} \Phi_{T}(u) d u, \\
D_{T} & =\frac{1}{2 \pi a_{c} \sigma_{c} \sqrt{T}} \int_{|u|>s_{T}}\left(1+\frac{i u}{a_{c} \sigma_{c} \sqrt{T}}\right)^{-1} \Phi_{T}(u) d u .
\end{aligned}
$$

From now on, we claim that for some positive constant $\nu$,

$$
\left|D_{T}\right|=\mathscr{O}\left(\exp \left(-\nu T^{1 / 3}\right)\right) .
$$

As a matter of fact, it follows from (6.1) that

$$
\left|\Phi_{T}(u)\right| \leqslant\left|\exp \left(T\left(L_{T}\left(a_{c}+\frac{i u}{\sigma_{c} \sqrt{T}}\right)-L_{T}\left(a_{c}\right)\right)\right)\right| .
$$

We also deduce from $(2.8)$ that $L\left(a_{c}\right)>0$ and thus, using Proposition 5 in Section 7.1 , we find that

$$
\left|\Phi_{T}(u)\right| \leqslant \exp \left(-T L\left(a_{c}\right)\right) \exp \left(-\frac{T u^{2}}{8 \varphi^{3}\left(a_{c}\right)}\left(1+\frac{4 u^{2}}{\varphi^{4}\left(a_{c}\right)}\right)^{-3 / 4}\right),
$$

which leads to (6.7). Finally, we deduce (4.8) from (6.2) and (6.5) together with standard calculus on the $\mathscr{N}(0,1)$ distribution.

6.2. Proof of Lemma 4. If $\Phi_{T}$ stands for the characteristic function of $U_{T}$ under $\mathbf{P}_{T}$, we have from (4.17)

$$
\Phi_{T}(u)=\exp \left(-i u c+T\left(L_{T}\left(a_{T}+\frac{i u}{T}\right)-L_{T}\left(a_{T}\right)\right)\right) .
$$

As in the proof of Lemma 2, it follows from Proposition 4 in Section 7.1 that for $T$ large enough, $\Phi_{T}$ belongs to $L^{2}(\mathbf{R})$. We shall now propose an asymptotic expansion for $\Phi_{T}$, slightly different from that of Lemma 7.2 of [3].

Lemma 8. For any $p>0$, there exist integers $q(p), r(p), s(p)$ and $a$ sequence $\left(\varphi_{k, l, m}^{H}\right)$ independent of $p$ such that for $T$ large enough

$$
\begin{aligned}
\Phi_{T}(u)= & \Phi(u) \exp \left(-\frac{\sigma_{H}^{2} u^{2}}{2 T}\right) \\
& \times\left[1+\sum_{k=1}^{p} \sum_{l=1}^{q(p)} \sum_{m=1}^{r(p)} \frac{\varphi_{k, l, m}^{H} u^{l}}{T^{k}\left(1-2 i \nu_{H} u\right)^{m}}+\mathscr{O}\left(\frac{\max \left(1,|u|^{s(p)}\right)}{T^{p+1}}\right)\right],
\end{aligned}
$$

where $\Phi$ is given by (4.31),

$$
\gamma_{H}=c-L^{\prime}\left(a_{H}\right)=\frac{1+2 \theta c \delta_{H}}{2 \theta \delta_{H}}, \quad \sigma_{H}^{2}=L^{\prime \prime}\left(a_{H}\right)=-\frac{1}{2 \theta^{3} \delta_{H}^{3}},
$$




$$
\nu_{H}=\frac{\left(1-\sin ^{2}(\pi H)\right) \gamma_{H}}{1-\sin ^{2}(\pi H)-(2 H-1)^{2} \sin (\pi H)\left(1+2 \theta c \delta_{H}\right)} .
$$

Moreover, the remainder $\mathscr{O}$ is uniform as soon as $|u| \leqslant s T^{2 / 3}$ for some positive constant $s$.

$\mathrm{R} \mathrm{e} \mathrm{m}$ a $\mathrm{rk} 5$. One can observe in this asymptotic expansion the limiting $\chi^{2}$ distribution $\Phi$ together with an independent centered Gaussian distribution with small variance $\sigma^{2} / T$.

P r o o f. First of all, we deduce from (4.9) that

$$
L_{T}\left(a_{T}\right)=L\left(a_{T}\right)+\frac{1}{T} H\left(a_{T}\right)+\frac{1}{T} K\left(a_{T}\right)+\frac{1}{T} \check{R}_{T}\left(a_{T}\right) .
$$

On the one hand, (2.8) implies that

$$
T\left(L\left(a_{T}+\frac{i u}{T}\right)-L\left(a_{T}\right)\right)=-\frac{T \varphi_{T}}{2}\left(\left(1-\frac{i u b_{T}}{T}\right)^{1 / 2}-1\right)
$$

with $b_{T}=2 / \varphi_{T}^{2}$. Consequently, for all $p \geqslant 2$

$$
\begin{aligned}
\exp & \left(T\left(L\left(a_{T}+\frac{i u}{T}\right)-L\left(a_{T}\right)\right)\right) \\
& =\exp \left(\frac{i u \varphi_{T} b_{T}}{4}-\frac{T \varphi_{T}}{2} \sum_{k=2}^{p} l_{k}\left(\frac{i u b_{T}}{T}\right)^{k}+\mathscr{O}\left(\frac{|u|^{p+1}}{T^{p}}\right)\right),
\end{aligned}
$$

where $l_{k}=-(2 k) ! /\left((2 k-1)\left(2^{k} k !\right)^{2}\right)$, which leads to

$$
\begin{aligned}
& \exp \left(-i u c+T\left(L\left(a_{T}+\frac{i u}{T}\right)-L\left(a_{T}\right)\right)\right) \\
& =\exp \left(-i u \gamma_{H}-\frac{\sigma_{H}^{2} u^{2}}{2 T}\right)\left[1+\sum_{k=1}^{p} \sum_{l=1}^{q(p)} \frac{\varphi_{k, l}^{H} u^{l}}{T^{k}}+\mathscr{O}\left(\frac{\max \left(1,|u|^{s(p)}\right)}{T^{p+1}}\right)\right]
\end{aligned}
$$

On the other hand, we also have from (2.9) that for all $p \geqslant 1$

$$
\begin{gathered}
\exp \left(H\left(a_{T}+\frac{i u}{T}\right)-H\left(a_{T}\right)\right)=\left(\frac{\varphi_{T}-\theta}{\varphi_{T}-\theta\left(1-i u b_{T} / T\right)^{-1 / 2}}\right)^{1 / 2} \\
=\left(1-\left(\frac{\theta}{\varphi_{T}-\theta}\right) \sum_{k=1}^{p} h_{k}\left(\frac{i u b_{T}}{T}\right)^{k}+\mathscr{O}\left(\frac{|u|^{p+1}}{T^{p+1}}\right)\right)^{-1 / 2}
\end{gathered}
$$

with $h_{k}=(2 k) ! /\left(2^{k} k !\right)^{2}$. Hence,

$$
\exp \left(H\left(a_{T}+\frac{i u}{T}\right)-H\left(a_{T}\right)\right)=\left[1+\sum_{k=1}^{p} \sum_{l=1}^{q(p)} \frac{\psi_{k, l}^{H} u^{l}}{T^{k}}+\mathscr{O}\left(\frac{\max \left(1,|u|^{s(p)}\right)}{T^{p+1}}\right)\right] .
$$


Furthermore, it follows from (4.10) that for all $p \geqslant 1$

$$
\begin{aligned}
\exp & \left(K\left(a_{T}+\frac{i u}{T}\right)-K\left(a_{T}\right)\right)=\left(\frac{2 \varphi_{T}+\left(\varphi_{T}+\theta\right) p_{H}}{2 \varphi_{T}+\varphi_{T} p_{H}+\theta p_{H}\left(1-i u b_{T} / T\right)^{-1 / 2}}\right)^{1 / 2} \\
= & \left(1+\left(\frac{\theta p_{H} T}{c_{T}}\right) \sum_{k=1}^{p} h_{k}\left(\frac{i u b_{T}}{T}\right)^{k}+\frac{1}{c_{T}} \mathscr{O}\left(\frac{|u|^{p+1}}{T^{p}}\right)\right)^{-1 / 2}
\end{aligned}
$$

where $c_{T}=T\left(2 \varphi_{T}+\left(\varphi_{T}+\theta\right) p_{H}\right)$. Therefore, if

$$
d_{T}(u)=1+\frac{i u \theta p_{H} b_{T}}{2 c_{T}}
$$

then we find that for all $p \geqslant 2$

$$
\begin{gathered}
\exp \left(K\left(a_{T}+\frac{i u}{T}\right)-K\left(a_{T}\right)\right) \\
=\frac{1}{\sqrt{d_{T}(u)}}\left(1+\left(\frac{\theta p_{H} T}{c_{T} d_{T}(u)}\right) \sum_{k=2}^{p} h_{k}\left(\frac{i u b_{T}}{T}\right)^{k}\right. \\
\left.\quad+\frac{1}{c_{T} d_{T}(u)} \mathscr{O}\left(\frac{|u|^{p+1}}{T^{p}}\right)\right)^{-1 / 2}
\end{gathered}
$$

One can easily check that as $T$ goes to infinity, the limits of $b_{T}, c_{T}$, and $d_{T}(u)$ are given by $2 /\left(\theta \delta_{H}\right)^{2},-\left(2+p_{H}\right) /\left(1+2 \theta c \delta_{H}\right)$, and $1-2 i \gamma_{H} u$, respectively, where $\gamma_{H}$ is given by (4.29). Then, we infer from (6.12) that for all $p \geqslant 2$

$$
\begin{aligned}
\exp \left(K\left(a_{T}+\frac{i u}{T}\right)-K\left(a_{T}\right)\right)=\frac{1}{\sqrt{1-2 i \gamma_{H} u}} \\
\quad \times\left[1+\sum_{k=1}^{p} \sum_{l=1}^{q(p)} \sum_{m=1}^{r(p)} \frac{\Psi_{k, l, m}^{H} u^{l}}{T^{k}\left(1-2 i \nu_{H} u\right)^{m}}+\mathscr{O}\left(\frac{\max \left(1,|u|^{s(p)}\right)}{T^{p+1}}\right)\right] .
\end{aligned}
$$

Now, in contrast with [3], the remainder term $\check{R}_{T}$ plays a prominent role that cannot be neglected. Let $\xi_{T}=T\left(\varphi_{T}+\theta\right)\left(r\left(a_{T}\right)-p_{H}\right) / c_{T}$ and

$$
\xi_{T}(u)=\frac{T}{c_{T}}\left(\varphi_{T}+\theta\left(1-\frac{i u b_{T}}{T}\right)^{-1 / 2}\right)\left(r_{T}\left(a_{T}+\frac{i u}{T}\right)-p_{H}\right) .
$$

One can observe that $\xi_{T}$ and $\xi_{T}(u)$ share the same limit

$$
\begin{aligned}
\lim _{T \rightarrow \infty} \xi_{T}(u)=\xi_{H} & =\frac{2\left(1-\delta_{H}\right)\left(1+2 \theta c \delta_{H}\right) r_{1}^{H}}{\delta_{H}\left(2+p_{H}\right) \sin (\pi H)} \\
& =-\frac{(2 H-1)^{2} \sin (\pi H)\left(1+2 \theta c \delta_{H}\right)}{1-\sin ^{2}(\pi H)} .
\end{aligned}
$$


In addition, it follows from (4.11) that

$$
\begin{aligned}
\exp \left(\check{R}_{T}\left(a_{T}\right)\right) & =\left(\frac{c_{T}}{c_{T}+c_{T} \xi_{T}}\right)^{1 / 2}\left[1+\mathscr{O}\left(T \exp \left(2 \theta T \delta_{H}\right)\right)\right] \\
& =\left(1+\xi_{T}\right)^{-1 / 2}\left[1+\mathscr{O}\left(T \exp \left(2 \theta T \delta_{H}\right)\right)\right] .
\end{aligned}
$$

Moreover, we also have

$$
\begin{aligned}
\exp \left(\check{R}_{T}\left(a_{T}+\frac{i u}{T}\right)\right)= & \left(\frac{d_{T}(u)+e_{T}(u)+\xi_{T}(u)}{d_{T}(u)+e_{T}(u)}\right)^{-1 / 2} \\
& \times\left[1+\mathscr{O}\left(T \exp \left(2 \theta T \delta_{H}\right)\right)\right]
\end{aligned}
$$

where

$$
e_{T}(u)=\frac{\theta p_{H} T}{c_{T}}\left(\left(1-\frac{i u b_{T}}{T}\right)^{-1 / 2}-1-\frac{i u b_{T}}{2 T}\right) .
$$

Therefore, via the same lines as in the proof of (6.13), we find that for all $p \geqslant 2$

$$
\begin{aligned}
\exp & \left(\check{R}_{T}\left(a_{T}+\frac{i u}{T}\right)-\check{R}_{T}\left(a_{T}\right)\right)=\frac{\sqrt{1-2 i \gamma_{H} u}}{\sqrt{1-2 i \nu_{H} u}} \\
& \times\left[1+\sum_{k=1}^{p} \sum_{l=1}^{q(p)} \sum_{m=1}^{r(p)} \frac{\Phi_{k, l, m}^{H} u^{l}}{T^{k}\left(1-2 i \nu_{H} u\right)^{m}}+\mathscr{O}\left(\frac{\max \left(1,|u|^{s(p)}\right)}{T^{p+1}}\right)\right]
\end{aligned}
$$

with

$$
\nu_{H}=\frac{\gamma_{H}}{1+\xi_{H}}=\frac{\left(1-\sin ^{2}(\pi H)\right) \gamma_{H}}{1-\sin ^{2}(\pi H)-(2 H-1)^{2} \sin (\pi H)\left(1+2 \theta c \delta_{H}\right)} .
$$

Finally, Lemma 8 follows from the conjunction of (6.10), (6.11), (6.13), and (6.14).

P r o o f of L e m m a 4 . Via Parseval's formula, $B_{T}$ given by (4.28) can be rewritten as

$$
B_{T}=\frac{1}{2 \pi T a_{T}} \int_{\mathbf{R}}\left(1+\frac{i u}{T a_{T}}\right)^{-1} \Phi_{T}(u) d u .
$$

Let $s_{T}>0$ be such that $\sqrt{T}=o\left(s_{T}\right)$ as $T$ goes to infinity. We can split $B_{T}=C_{T}+D_{T}$, where

$$
\begin{aligned}
C_{T} & =\frac{1}{2 \pi T a_{T}} \int_{|u| \leqslant s_{T}}\left(1+\frac{i u}{T a_{T}}\right)^{-1} \Phi_{T}(u) d u, \\
D_{T} & =\frac{1}{2 \pi T a_{T}} \int_{|u|>s_{T}}\left(1+\frac{i u}{T a_{T}}\right)^{-1} \Phi_{T}(u) d u .
\end{aligned}
$$


On the one hand, we find from Proposition 4 , and the fact that $x \mapsto x(1+$ $x)^{-3 / 4}$ is increasing that for some positive constant $\mu$,

$$
\left|D_{T}\right|=\mathscr{O}\left(T\left(1+T^{3 / 2}\right) \exp \left(-\frac{\mu s_{T}^{2}}{T}\left(1+\frac{s_{T}^{2}}{T^{2}}\right)^{-3 / 4}\right)\right) .
$$

It clearly leads to

$$
\left|D_{T}\right|=\mathscr{O}\left(\exp \left(-\frac{\mu s_{T}^{2}}{T}\right)\right)
$$

On the other hand, the asymptotic expansion for $C_{T}$, which immediately leads to (4.32), follows from Lemma 14, completing the proof of Lemma 4.

6.3. Proof of Lemma 5. The proof follows the same lines as the proof of Lemma 4. The most important difference is that the scale of Taylor expansion is in $\sqrt{T}$ instead of $T$. Since $\Phi_{T}$ is the characteristic function of $U_{T}$ defined by (4.38) under $\mathbf{P}_{T}$ defined by (4.17), we have

$$
\Phi_{T}(u)=\exp \left(\frac{i u \sqrt{T}}{2 \theta \delta_{H}}+T\left(L_{T}\left(a_{T}+\frac{i u}{\sqrt{T}}\right)-L_{T}\left(a_{T}\right)\right)\right) .
$$

As in the proof of Lemma 2, it follows from Proposition 4 in Section 7.1 that for $T$ large enough $\Phi_{T}$ belongs to $L^{2}(\mathbf{R})$. We shall now propose an asymptotic expansion for $\Phi_{T}$ slightly different from that of Lemma 8.

Lemma 9. For any $p>0$, there exist integers $q(p), r(p), s(p)$ and a sequence $\left(\varphi_{k, l, m}^{H}\right)$ independent of $p$ such that for $T$ large enough

$$
\Phi_{T}(u)=\Phi(u)\left[1+\sum_{k=1}^{p} \sum_{l=1}^{q(p)} \sum_{m=1}^{r(p)} \frac{\varphi_{k, l, m}^{H} u^{l}}{(\sqrt{T})^{k}\left(1-2 i \eta_{H} u\right)^{m}}+\mathscr{O}\left(\frac{\max \left(1,|u|^{s(p)}\right)}{(\sqrt{T})^{p+1}}\right)\right]
$$

where $\Phi$ is given by (4.41). Moreover, the remainder $\mathscr{O}$ is uniform as soon as $|u| \leqslant s T^{1 / 6}$ for some positive constant $s$.

P r o o f. First of all, we deduce from (4.9) that

$$
L_{T}\left(a_{T}\right)=L\left(a_{T}\right)+\frac{1}{T} H\left(a_{T}\right)+\frac{1}{T} K\left(a_{T}\right)+\frac{1}{T} \check{R}_{T}\left(a_{T}\right) .
$$

On the one hand, (2.8) implies that

$$
T\left(L\left(a_{T}+\frac{i u}{\sqrt{T}}\right)-L\left(a_{T}\right)\right)=-\frac{T \varphi_{T}}{2}\left(\left(1-\frac{i u b_{T}}{\sqrt{T}}\right)^{1 / 2}-1\right)
$$

with $b_{T}=2 / \varphi_{T}^{2}$. Consequently, for all $p \geqslant 2$

$$
\begin{aligned}
\exp & \left(T\left(L\left(a_{T}+\frac{i u}{\sqrt{T}}\right)-L\left(a_{T}\right)\right)\right) \\
& =\exp \left(\frac{i u \varphi_{T} b_{T} \sqrt{T}}{4}-\frac{T \varphi_{T}}{2} \sum_{k=2}^{p} l_{k}\left(\frac{i u b_{T}}{\sqrt{T}}\right)^{k}+\mathscr{O}\left(\frac{|u|^{p+1}}{(\sqrt{T})^{p+1}}\right)\right),
\end{aligned}
$$


where $l_{k}=-(2 k) ! /\left((2 k-1)\left(2^{k} k !\right)^{2}\right)$, which leads to

$$
\begin{gathered}
\exp \left(\frac{i u \sqrt{T}}{2 \theta \delta_{H}}+T\left(L\left(a_{T}+\frac{i u}{\sqrt{T}}\right)-L\left(a_{T}\right)\right)\right)=\exp \left(-i u \eta_{H}-\frac{u^{2} \sigma_{H}^{2}}{2}\right) \\
\times\left[1+\sum_{k=1}^{p} \sum_{l=1}^{q(p)} \frac{\varphi_{k, l}^{H} u^{l}}{(\sqrt{T})^{k}}+\mathscr{O}\left(\frac{\max \left(1,|u|^{s(p)}\right)}{(\sqrt{T})^{p+1}}\right)\right] .
\end{gathered}
$$

On the other hand, we also have from (2.9) that for all $p \geqslant 1$

$$
\begin{gathered}
\exp \left(H\left(a_{T}+\frac{i u}{\sqrt{T}}\right)-H\left(a_{T}\right)\right)=\left(\frac{\varphi_{T}-\theta}{\varphi_{T}-\theta\left(1-i u b_{T} / \sqrt{T}\right)^{-1 / 2}}\right)^{1 / 2} \\
=\left(1-\left(\frac{\theta}{\varphi_{T}-\theta}\right) \sum_{k=1}^{p} h_{k}\left(\frac{i u b_{T}}{\sqrt{T}}\right)^{k}+\mathscr{O}\left(\frac{|u|^{p+1}}{(\sqrt{T})^{p+1}}\right)\right)^{-1 / 2}
\end{gathered}
$$

with $h_{k}=(2 k) ! /\left(2^{k} k !\right)^{2}$. Hence,

$$
\exp \left(H\left(a_{T}+\frac{i u}{\sqrt{T}}\right)-H\left(a_{T}\right)\right)=1+\sum_{k=1}^{p} \sum_{l=1}^{q(p)} \frac{\psi_{k, l}^{H} u^{l}}{(\sqrt{T})^{k}}+\mathscr{O}\left(\frac{\max \left(1,|u|^{s(p)}\right)}{(\sqrt{T})^{p+1}}\right) .
$$

Furthermore, it follows from (4.10) that for all $p \geqslant 1$

$$
\begin{aligned}
\exp & \left(K\left(a_{T}+\frac{i u}{\sqrt{T}}\right)-K\left(a_{T}\right)\right) \\
& =\left(\frac{2 \varphi_{T}+\left(\varphi_{T}+\theta\right) p_{H}}{2 \varphi_{T}+\varphi_{T} p_{H}+\theta p_{H}\left(1-i u b_{T} / \sqrt{T}\right)^{-1 / 2}}\right)^{1 / 2} \\
& =\left(1+\left(\frac{\theta p_{H} \sqrt{T}}{c_{T}}\right) \sum_{k=1}^{p} h_{k}\left(\frac{i u b_{T}}{\sqrt{T}}\right)^{k}+\frac{1}{c_{T}} \mathscr{O}\left(\frac{|u|^{p+1}}{(\sqrt{T})^{p}}\right)\right)^{-1 / 2},
\end{aligned}
$$

where $c_{T}=\sqrt{T}\left(2 \varphi_{T}+\left(\varphi_{T}+\theta\right) p_{H}\right)$. Therefore, if $d_{T}(u)=1+i u \theta p_{H} b_{T} /\left(2 c_{T}\right)$, then we find that for all $p \geqslant 2$

$$
\begin{aligned}
\exp & \left(K\left(a_{T}+\frac{i u}{\sqrt{T}}\right)-K\left(a_{T}\right)\right)=\frac{1}{\sqrt{d_{T}(u)}} \\
& \times\left(1+\frac{\theta p_{H} T}{c_{T} d_{T}(u)} \sum_{k=2}^{p} h_{k}\left(\frac{i u b_{T}}{\sqrt{T}}\right)^{k}+\frac{1}{c_{T} d_{T}(u)} \mathscr{O}\left(\frac{|u|^{p+1}}{(\sqrt{T})^{p}}\right)\right)^{-1 / 2} .
\end{aligned}
$$

One can easily check that as $T$ goes to infinity, the limits of $b_{T}, c_{T}$, and $d_{T}(u)$ are given by $2 /\left(\theta \delta_{H}\right)^{2},\left(2+p_{H}\right) \sqrt{-\theta \delta_{H}}$, and $1-2 i \eta_{H} u$, respectively, where $\eta_{H}$ is given by (4.40). Then, we infer from (6.22) that for all $p \geqslant 2$

$$
\begin{aligned}
\exp & \left(K\left(a_{T}+\frac{i u}{\sqrt{T}}\right)-K\left(a_{T}\right)\right)=\frac{1}{\sqrt{1-2 i \eta_{H} u}} \\
& \times\left[1+\sum_{k=1}^{p} \sum_{l=1}^{q(p)} \sum_{m=1}^{r(p)} \frac{\Psi_{k, l, m}^{H} u^{l}}{(\sqrt{T})^{k}\left(1-2 i \eta_{H} u\right)^{m}}+\mathscr{O}\left(\frac{\max \left(1,|u|^{s(p)}\right)}{(\sqrt{T})^{p+1}}\right)\right] .
\end{aligned}
$$


Now, in contrast with [3], the remainder term $\check{R}_{T}$ plays a prominent role that cannot be neglected. Let $\xi_{T}=\sqrt{T}\left(\varphi_{T}+\theta\right)\left(r\left(a_{T}\right)-p_{H}\right) / c_{T}$ and

$$
\xi_{T}(u)=\frac{\sqrt{T}}{c_{T}}\left(\varphi_{T}+\theta\left(1-\frac{i u b_{T}}{\sqrt{T}}\right)^{-1 / 2}\right)\left(r_{T}\left(a_{T}+\frac{i u}{\sqrt{T}}\right)-p_{H}\right) .
$$

One can observe that $\xi_{T}$ and $\xi_{T}(u)$ share the same limit

$$
\lim _{T \rightarrow \infty} \xi_{T}(u)=0 .
$$

In addition, it follows from (4.11) that

$$
\begin{aligned}
\exp \left(\check{R}_{T}\left(a_{T}\right)\right) & =\left(\frac{c_{T}}{c_{T}+c_{T} \xi_{T}}\right)^{1 / 2}\left[1+\mathscr{O}\left(\sqrt{T} \exp \left(2 \theta T \delta_{H}\right)\right)\right] \\
& =\left(1+\xi_{T}\right)^{-1 / 2}\left[1+\mathscr{O}\left(\sqrt{T} \exp \left(2 \theta T \delta_{H}\right)\right)\right] .
\end{aligned}
$$

Moreover, we also have

$$
\begin{aligned}
\exp \left(\check{R}_{T}\left(a_{T}+\frac{i u}{T}\right)\right)= & \left(\frac{d_{T}(u)+e_{T}(u)+\xi_{T}(u)}{d_{T}(u)+e_{T}(u)}\right)^{-1 / 2} \\
& \times\left[1+\mathscr{O}\left(\sqrt{T} \exp \left(2 \theta T \delta_{H}\right)\right)\right]
\end{aligned}
$$

where

$$
e_{T}(u)=\frac{\theta p_{H} T}{c_{T}}\left(\left(1-\frac{i u b_{T}}{\sqrt{T}}\right)^{-1 / 2}-1-\frac{i u b_{T}}{2 \sqrt{T}}\right) .
$$

Therefore, via the same lines as in the proof of relation (6.23), we find that for all $p \geqslant 2$

$$
\begin{aligned}
\exp & \left(\check{R}_{T}\left(a_{T}+\frac{i u}{T}\right)-\check{R}_{T}\left(a_{T}\right)\right) \\
& =1+\sum_{k=1}^{p} \sum_{l=1}^{q(p)} \sum_{m=1}^{r(p)} \frac{\Phi_{k, l, m}^{H} u^{l}}{(\sqrt{T})^{k}\left(1-2 i \eta_{H} u\right)^{m}}+\mathscr{O}\left(\frac{\max \left(1,|u|^{s(p)}\right)}{(\sqrt{T})^{p+1}}\right) .
\end{aligned}
$$

Finally, Lemma 9 follows from the conjunction of (6.20), (6.21), (6.23), and (6.24).

P r o of of $\mathrm{L}$ e $\mathrm{m} \mathrm{m}$ a 5 . Via Parseval's formula, $B_{T}$ given by (4.28) can be rewritten as

$$
B_{T}=\frac{1}{2 \pi T a_{T}} \int_{\mathbf{R}}\left(1+\frac{i u}{T a_{T}}\right)^{-1} \Phi_{T}(u) d u .
$$

For some positive constant $s$, set $s_{T}=s T^{1 / 6}$. We can split $B_{T}=C_{T}+D_{T}$, where

$$
\begin{aligned}
C_{T} & =\frac{1}{2 \pi T a_{T}} \int_{|u| \leqslant s_{T}}\left(1+\frac{i u}{T a_{T}}\right)^{-1} \Phi_{T}(u) d u, \\
D_{T} & =\frac{1}{2 \pi T a_{T}} \int_{|u|>s_{T}}\left(1+\frac{i u}{T a_{T}}\right)^{-1} \Phi_{T}(u) d u .
\end{aligned}
$$


On the one hand, we find from Proposition 4 that for some constant $\mu>0$,

$$
\left|D_{T}\right|=\mathscr{O}\left(\exp \left(-\frac{\mu s_{T}^{2}}{T}\right)\right)
$$

On the other hand, the asymptotic expansion for $C_{T}$, which immediately leads to (4.32), follows from the same arguments as those in [3, Section 7.4].

\section{Appendix C: Technical results.}

7.1. Statement of the results. The main interest of the decomposition (4.9) is given by the two following results. They show us that the different functions we deal with are holomorphic and that the behavior of the remainder is negligible in our calculations.

Proposition 3. Denote

$$
\begin{aligned}
& \mathscr{D}_{\Delta}=\left\{z \in \mathbf{C}: \operatorname{Re} z<a_{H}\right\}, \\
& \mathscr{D}_{1}=\left\{z \in \mathbf{C}: \operatorname{Re} z<a_{H}-\varepsilon(2+\varepsilon) \frac{\theta^{2} \delta_{H}^{2}}{2}\right\}, \quad \varepsilon>0 .
\end{aligned}
$$

Then, for $T$ large enough, we have the following assertions.

a) The functions $\varphi, L_{T}, L, H, K, \check{R}_{T}$ have analytic extensions to $\mathscr{D}_{\Delta}$.

b) The function $(z, T) \mapsto \check{R}_{T}(z)$ is $C^{\infty}$ on $\mathscr{D}_{\Delta} \times\left[T_{\Delta},+\infty\left[\right.\right.$, for $T_{\Delta}$ depending only on $H$ and $\theta$.

c) For $\varepsilon>0$, and for all $z \in \mathscr{D}_{1}$,

$$
\sqrt{\frac{\delta_{H}}{\left(2+p_{H}\right)\left(\delta_{H}+1\right)}} \leqslant|\exp (H(z)+K(z))| \leqslant \frac{4}{\sqrt{2+p_{H}}} \sqrt{\frac{1+\varepsilon}{\varepsilon}} .
$$

d) There exists a constant $C$ depending only on $\theta$ and $H$ such that for $T$ large enough and for all $z \in \mathscr{D}_{1}$,

$$
\sqrt{\frac{1}{2}-\frac{C}{T^{2} \varepsilon\left(\delta_{H} \theta\right)^{4}}} \leqslant\left|\exp \left(-\check{R}_{T}(z)\right)\right| \leqslant C \sqrt{1+\frac{1}{T}+\frac{1}{T \varepsilon}} .
$$

Proposition 4. For $T$ large enough, $\varepsilon>2 C /\left(T^{2}\left(\delta_{H} \theta\right)^{4}\right), a \in \mathscr{D}_{1} \cap \mathbf{R}$, and $u \in \mathbf{R}$, we have

$$
\begin{aligned}
& \left|\exp \left(T\left(L_{T}(a+i u)-L_{T}(a)\right)\right)\right| \\
& \quad \leqslant C\left(1+T^{3 / 2}\right) \exp \left(-\frac{T u^{2}}{8 \varphi^{3}(a)}\left(1+\frac{4 u^{2}}{\varphi^{4}(a)}\right)^{-3 / 4}\right)
\end{aligned}
$$

and the map $u \mapsto \exp \left(T\left(L_{T}(a+i u)-L_{T}(a)\right)\right)$ belongs to $L^{2}(\mathbf{R})$.

Proposition 5. Let $a \in \mathbf{R}$ be such that $a<a_{H}$. Then, as $T$ goes to infinity, we have

$$
\exp \left(-\check{R}_{T}(a)\right)=\mathscr{O}\left(\max \left\{1 ; \frac{-1}{T\left(\varphi(a)+\delta_{H} \theta\right)}\right\}\right)
$$


7.2. Proofs of the results. We shall denote the principal determination of the logarithm defined on $\mathbf{C} \backslash]-\infty, 0]$ by

$$
\ln z=\ln |z|+i \operatorname{Arg}(z),
$$

where

$$
\operatorname{Arg}(z)= \begin{cases}\arcsin \left(|z|^{-1} \operatorname{Im} z\right) & \text { if } \operatorname{Re} z \geqslant 0 \\ \arccos \left(|z|^{-1} \operatorname{Re} z\right) & \text { if } \operatorname{Re} z<0, \operatorname{Im} z>0 \\ -\arccos \left(|z|^{-1} \operatorname{Re} z\right) & \text { if } \operatorname{Re} z<0, \operatorname{Im} z<0\end{cases}
$$

Pro of of Proposition 3 . Since $T \mapsto S_{T}$ is a positive increasing process, we have for $a<a_{H}$

$$
\mathbf{E}\left[\exp \left(a S_{T}\right)\right] \leqslant \lim _{T \rightarrow+\infty} \exp \left(T L_{T}(a)\right)<\infty .
$$

The Lebesgue dominated convergence theorem yields that $z \mapsto \mathbf{E}\left[\exp \left(z S_{T}\right)\right]$ has an analytic extension to $\left\{z \in \mathbf{C}, \operatorname{Re} z<a_{H}\right\}$. In order to prove Proposition 3, we have to obtain the same result for $\varphi, L, H, K$, and $\check{R}_{T}$. The proof is split in steps. First, we study the function $\varphi$.

Lemma 10. The function $\varphi$ has an analytic extension on $\{z \in$ $\left.\mathbf{C}, \operatorname{Re} z<a_{H}\right\}$ still denoted by $\varphi$ such that $\left.\operatorname{Arg}(\varphi) \in\right]-\pi / 4, \pi / 4[, \operatorname{Re} \varphi \in$ ]$-\delta_{H} \theta,+\infty[, \operatorname{Im} \varphi(z)$ vanishes if and only if $\operatorname{Im} z=0$, and for $\varepsilon>0$,

$$
\inf _{z \in \mathscr{D}_{1}} \operatorname{Re} \varphi(z)>-\theta \delta_{H}(1+\varepsilon) .
$$

For all $a<a_{H}$ and $u \in \mathbf{R}$,

$$
\operatorname{Re}(\varphi(a+i u)-\varphi(a)) \geqslant \frac{u^{2}}{2 \varphi^{3}(a)}\left(1+\frac{4 u^{2}}{\varphi^{4}(a)}\right)^{-3 / 4} .
$$

P r o of of $\mathrm{L}$ e m m a 10. The properties of $\varphi$ relies on the properties of the analytic function defined for all $z \in \mathbf{C}, \operatorname{Re} z>0$, by

$$
\sqrt{1+z}=\sqrt{|1+z|} \exp \left(\frac{i}{2} \operatorname{Arg}(1+z)\right) .
$$

On the one hand, the imaginary part of $\sqrt{1+z}$ is given by

$$
\operatorname{Im} \sqrt{1+z}=\frac{\sqrt{|1+z|-\operatorname{Re}(1+z)}}{\sqrt{2}} \operatorname{sign}(\operatorname{Im} z) .
$$

If $\operatorname{Re} z>0$, then $\operatorname{Arg}(1+z)$ belongs to $]-\pi / 2, \pi / 2[$, and $\operatorname{Arg}(\sqrt{1+z})$ belongs to $]-\pi / 4, \pi / 4$. On the other hand, the real part of $\sqrt{1+z}$ is given by

$$
\operatorname{Re} \sqrt{1+z}=\frac{\sqrt{|1+z|+\operatorname{Re}(1+z)}}{\sqrt{2}} .
$$


If $\operatorname{Re} z>0$, then $|1+z| \geqslant|\operatorname{Re}(1+z)|=\operatorname{Re}(1+z)$ and (7.2) leads us to

$$
\operatorname{Re} \sqrt{1+z} \geqslant \sqrt{\operatorname{Re}(1+z)} .
$$

Thus, for all $z \in \mathbf{C}$ such that $\operatorname{Re} z>\varepsilon(2+\varepsilon)$ we have $\operatorname{Re}(1+z)>(1+\varepsilon)^{2}$ which clearly implies that

$$
\operatorname{Re}(\sqrt{1+z}) \geqslant 1+\varepsilon
$$

Now, by the very definition of $\varphi$, we have

$$
\varphi(z)=-\delta_{H} \theta \sqrt{1+\frac{2}{\delta_{H}^{2} \theta^{2}}\left(a_{H}-z\right)} .
$$

Consequently, the condition $z \in \mathscr{D}_{1}$ leads to $2 \delta_{H}^{-2} \theta^{-2} \operatorname{Re}\left(a_{H}-z\right)>\varepsilon(2+\varepsilon)$ and thus

$$
\inf _{z \in \mathscr{D}_{1}} \operatorname{Re} \varphi(z)>-\theta \delta_{H}(1+\varepsilon) .
$$

Futhermore, we have, for all $a<a_{H}$ and $u \in \mathbf{R}$,

$$
\operatorname{Re}(\varphi(a+i u)-\varphi(a))=\varphi(a) \operatorname{Re}\left(\sqrt{1-\frac{2 i u}{\varphi^{2}(a)}}-1\right) .
$$

Therefore, it follows from relation (7.2) that

$$
\operatorname{Re}(\varphi(a+i u)-\varphi(a))=\frac{\varphi(a)}{\sqrt{2}}\left(\sqrt{\sqrt{1+\frac{4 u^{2}}{\varphi^{4}(a)}}+1}-\sqrt{2}\right) .
$$

Finally, inequality (7.1) follows from the fact that, for any $x \geqslant 0$,

$$
\sqrt{\sqrt{1+x}+1}-\sqrt{2} \geqslant \frac{x}{4 \sqrt{2}(1+x)^{3 / 4}}
$$

which is applied to $x=4 u^{2} / \varphi^{4}(a)$.

Hereafter, we study $H$ and $K$. Observe that if $a \in]-\infty, 0]$, then $H(a)=$ $\widetilde{H}(\varphi(a))$ and $K(a)=\widetilde{K}(\varphi(a))$, where for $z \in \mathbf{C}$ such that $\operatorname{Re} z>-\delta_{H} \theta$ the functions $\widetilde{H}$ and $\widetilde{K}$ are given by

$$
\widetilde{H}(z)=-\frac{1}{2} \ln \frac{z-\theta}{2 z}, \quad \widetilde{K}(z)=-\frac{1}{2} \ln \left(1+\frac{z+\theta}{2 z} p_{H}\right) .
$$

Then, the expected properties of $H$ and $K$ are some consequences of Lemma 10 and the same properties of $\widetilde{H}$ and $\widetilde{K}$ on $\left\{z \in \mathbf{C}: \operatorname{Re} z>-\delta_{H} \theta\right\}$ instead of $\left\{z \in \mathbf{C}: \operatorname{Re} z<a_{H}\right\}$. 
Lemma 11. The functions $\widetilde{H}$ and $\widetilde{K}$ admit analytic extensions on $\{z \in$ C: $\left.\operatorname{Re} z>-\delta_{H} \theta\right\}$. Moreover, if for all $\varepsilon>0$, we denote

$$
\mathscr{D}_{2}=\left\{z \in \mathbf{C}: \operatorname{Re} z>-\delta_{H} \theta(1+\varepsilon),|\operatorname{Arg}(z)| \leqslant \frac{\pi}{4}\right\},
$$

then for $z \in \mathscr{D}_{2}$

$$
\sqrt{\frac{\delta_{H}}{\left(2+p_{H}\right)\left(\delta_{H}+1\right)}} \leqslant|\exp (\widetilde{H}(z)+\widetilde{K}(z))| \leqslant \frac{4}{\sqrt{2+p_{H}}} \sqrt{\frac{1+\varepsilon}{\varepsilon}} .
$$

$\mathrm{P}$ r o of of $\mathrm{L}$ e $\mathrm{m} \mathrm{m}$ a 11 . Using (7.4), it is easy to prove the analytic extensions of $\widetilde{H}$ and $\widetilde{K}$ on $\left\{z \in \mathbf{C}: \operatorname{Re} z>-\delta_{H} \theta\right\}$. For $\widetilde{K}$ it is a consequence of the fact that for $z \in \mathbf{C}, \operatorname{Re} z>-\delta_{H} \theta$,

$$
\operatorname{Re}\left(1+\frac{z+\theta}{2 z} p_{H}\right) \geqslant 1+\frac{p_{H}}{2}+\frac{\theta p_{H}}{2 \operatorname{Re} z}>1+\frac{p_{H}}{2}-\frac{p_{H}}{2 \delta_{H}}=0 .
$$

It remains to prove the inequalities stated in the lemma. We have

$$
\begin{aligned}
\widetilde{H}(z)+\widetilde{K}(z) & =-\frac{1}{2}\left(\ln (z-\theta)+\ln \left(2 z+(z+\theta) p_{H}\right)-2 \ln (2 z)\right) \\
& =-\frac{1}{2}\left(\ln (z-\theta)+\ln \left(\left(2+p_{H}\right) z+\theta p_{H}\right)-2 \ln (2 z)\right) \\
& =-\frac{1}{2}\left(\ln (z-\theta)+\ln \left(2+p_{H}\right)+\ln \left(z+\frac{\theta p_{H}}{2+p_{H}}\right)-2 \ln (2 z)\right) \\
& =-\frac{1}{2}\left(\ln \left(2+p_{H}\right)+\ln (z-\theta)+\ln \left(z+\delta_{H} \theta\right)-\ln 4-2 \ln z\right) .
\end{aligned}
$$

Thus

$$
\exp (\widetilde{H}(z)+\widetilde{K}(z))=\left(\frac{\left(2+p_{H}\right)(z-\theta)\left(z+\theta \delta_{H}\right)}{4 z^{2}}\right)^{-1 / 2},
$$

where $\sqrt{z}=\sqrt{|z|} \exp (i \operatorname{Arg}(z) / 2)$. For $z \in \mathbf{C}$ such that $|\operatorname{Arg}(z)| \leqslant \pi / 4$, we have $|\operatorname{Im} z| \leqslant \operatorname{Re} z$; if moreover we have $\operatorname{Re} z>-\delta_{H} \theta(1+\varepsilon)$, then

$$
\begin{gathered}
1 \geqslant\left|\frac{z+\delta_{H} \theta}{z}\right| \geqslant \frac{\operatorname{Re} z+\delta_{H} \theta}{\operatorname{Re} z} \geqslant \frac{\varepsilon}{(1+\varepsilon)}, \\
\frac{1+\delta_{H}}{\delta_{H}} \geqslant\left|\frac{z-\theta}{z}\right| \geqslant \frac{\operatorname{Re} z-\theta}{2 \operatorname{Re} z} \geqslant 1 .
\end{gathered}
$$

We have used the fact that the function $x \mapsto\left(x+\delta_{H} \theta\right) / x$ is increasing and the function $x \mapsto(x-\theta) / x$ is decreasing. Assertion (c) of Proposition 3 is then proved. 
Now, we focus on $\check{R}_{T}$. First, observe that for $z \in \mathbf{C}$ such that $\operatorname{Re} z<0$, we have $\check{R}_{T}(z)=\widetilde{R}_{T}(\varphi(z))$, where for $z \in \mathbf{C}$ such that $\operatorname{Re} z>-\delta_{H} \theta$,

$$
\begin{aligned}
\widetilde{R}_{T}(z)=-\frac{1}{2} \ln \left[1+\frac{(z+\theta)\left(\widetilde{r}_{T}(z)-p_{H}\right)}{\left(2+p_{H}\right)\left(z+\delta_{H} \theta\right)}+\frac{(z+\theta)^{2}}{\left(2+p_{H}\right)(z-\theta)\left(z+\delta_{H} \theta\right)} e^{-2 T z}\right] \\
=-\frac{1}{2} \ln \left[z\left(2+p_{H}\right)\left(z+\delta_{H} \theta\right)+z(z+\theta)\left(\tilde{r}_{T}(z)-p_{H}\right)\right. \\
\left.\quad+\frac{z(z+\theta)^{2}}{(z-\theta)} e^{-2 T z}\right]+\frac{1}{2} \ln \left[z\left(2+p_{H}\right)\left(z+\delta_{H} \theta\right)\right] \\
=-\frac{1}{2}\left(\ln \left[\widetilde{\widetilde{R}}_{1, T}(z)\right]-\ln \left[\left(2+p_{H}\right) z\right]-\ln \left[z+\delta_{H} \theta\right]\right)
\end{aligned}
$$

where

$$
\begin{aligned}
\tilde{r}_{T}(z) & =r_{H}\left(\frac{T z}{2}\right) e^{-T z}-1, \\
\widetilde{R}_{1, T}(z) & =z\left(2+p_{H}\right)\left(z+\delta_{H} \theta\right)+z(z+\theta)\left(\tilde{r}_{T}(z)-p_{H}\right)+\frac{z(z+\theta)^{2}}{z-\theta} e^{-2 T z} .
\end{aligned}
$$

The properties of $\check{R}_{T}$ are some consequences of Lemma 10 and the following lemma.

Lemma 12. Denote

$$
\mathscr{D}_{3}=\left\{z \in \mathbf{C}: \operatorname{Re} z>-\delta_{H} \theta, \operatorname{Arg}(z) \in\right]-\frac{\pi}{4}, \frac{\pi}{4}[\} .
$$

For $T$ large enough and for all $\left.\left.z \in \mathscr{D}_{3}, \widetilde{\widetilde{R}}_{1, T}(z) \in \mathbf{C} \backslash\right]-\infty, 0\right]$.

In fact, Lemma 12 and decomposition (7.6) give us an analytic extension of $\widetilde{\widetilde{R}}_{T}$ on $\mathscr{D}_{3}$. Moreover, there exists $T_{3}$ depending only on $H$ and $\theta$ such that the function

$$
(z, T) \mapsto \frac{\widetilde{\widetilde{R}}_{1, T}(z)}{\left(2+p_{H}\right) z\left(z+\delta_{H} \theta\right)}
$$

is $C^{\infty}$ and never vanishes on $\left\{(z, T): z \in \mathscr{D}_{3}, T>T_{3}\right\}$ and is $C^{\infty}$ with respect to $(z, T)$. Since $\check{R}_{T}=\widetilde{\mathscr{R}}_{T}(\varphi)$, the function $(z, T) \mapsto \check{R}_{T}(z)$ is $C^{\infty}$ on $\left\{T>T_{3}\right\}$ and $\left\{z \in \mathbf{C}: \operatorname{Re} z<a_{H}\right\}$. Assertion (b) of Proposition 3 is then proved.

P r o of of $\mathrm{L}$ e m m a 12 . We recall that for $z \in \mathbf{C}$ such that $\operatorname{Arg}(z) \in]-\pi / 4, \pi / 4[$

$$
r_{H}(z)-1=\frac{\exp (2 z)}{\sin (\pi H)}\left(1-\frac{(2 H-1)^{2}}{4 z}+\frac{1}{z^{2}} \sin (\pi H) F(z)\right),
$$

where $F$ is a continuous bounded function. Then, for $z \in \mathscr{D}_{3}$,

$$
\tilde{r}_{T}(z)-p_{H}=\frac{r_{1}}{T z}+\frac{1}{T^{2} z^{2}} F(T z) .
$$


We can exhibit a polynomial term from $\widetilde{\mathscr{R}}_{1, T}(z)$ :

$$
\begin{aligned}
\widetilde{R}_{1, T}(z)= & z\left(2+p_{H}\right)\left(z+\delta_{H} \theta\right) \\
& +z(z+\theta)\left(\frac{r_{1}}{T z}+\frac{1}{T^{2} z^{2}} F(T z)\right)+\frac{z(z+\theta)^{2}}{z-\theta} e^{-2 T z} \\
= & z\left(2+p_{H}\right)\left(z+\delta_{H} \theta\right)+(z+\theta) \frac{r_{1}}{T} \\
& +\left(\frac{z+\theta}{T^{2} z} F(T z)+\frac{z(z+\theta)^{2}}{z-\theta} e^{-2 T z}\right) \\
= & z\left(2+p_{H}\right)\left(z+\delta_{H} \theta\right)+(z+\theta) \frac{r_{1}}{T} \\
& +\frac{z+\theta}{T^{2} z}\left(F(T z)+\frac{T^{2} z^{2}(z+\theta)}{z-\theta} e^{-2 T z}\right) .
\end{aligned}
$$

Let us denote

$$
\begin{aligned}
\widetilde{P}_{T}(z) & =z\left(2+p_{H}\right)\left(z+\delta_{H} \theta\right)+(z+\theta) \frac{r_{1}}{T}, \\
C & =\frac{1-\delta_{H}}{\delta_{H}} \sup _{z \in \mathscr{D}_{3}}\left|F(T z)+\frac{z+\theta}{z-\theta} T^{2} z^{2} e^{-2 z T}\right|<+\infty .
\end{aligned}
$$

Observe that for $z \in \mathscr{D}_{3}$, on the one hand,

$$
\begin{aligned}
\left|\operatorname{Im} \widetilde{P}_{T}(z)\right| & =|\operatorname{Im} z|\left[\left(2+p_{H}\right)\left(2 \operatorname{Re} z+\delta_{H} \theta\right)+\frac{r_{1}}{T}\right] \\
& \geqslant|\operatorname{Im} z|\left[\frac{2+p_{H}}{2}\left(-\delta_{H} \theta\right)+\frac{r_{1}}{T}\right] .
\end{aligned}
$$

Then, for $z \in \mathbf{C}$ such that

$$
\operatorname{Im} z>\frac{4}{2+p_{H}} \frac{1}{\left(-\delta_{H} \theta\right)}\left[\frac{C}{T^{2}}-\frac{r_{1}}{T}\right]
$$

we have $\left|\operatorname{Im} \widetilde{R}_{1, T}(z)\right|>0$. On the other hand,

$$
\begin{aligned}
\operatorname{Re} \widetilde{P}_{T}(z) & =\left(2+p_{H}\right) \operatorname{Re}(z)\left(\operatorname{Re} z+\delta_{H} \theta\right)+\frac{\theta r_{1}}{T} \operatorname{Re} z-\left(2+p_{H}\right)(\operatorname{Im} z)^{2}, \\
& \geqslant-\delta_{H} \theta^{2} \frac{r_{1}}{T}-\left(2+p_{H}\right)(\operatorname{Im} z)^{2} .
\end{aligned}
$$

Then, for $T$ large enough and for all $z \in \mathscr{D}_{3}$ such that

$$
\operatorname{Im} z>\frac{4}{2+p_{H}} \frac{1}{\left(-\delta_{H} \theta\right)}\left[\frac{C}{T^{2}}-\frac{r_{1}}{T}\right]
$$

we have $\operatorname{Re} \widetilde{\mathscr{R}}_{1, T}(z)>0$. We are allowed to conclude that if $T$ is large enough and $z \in \mathscr{D}_{3}$, then $\left.\left.\widetilde{R}_{1, T}(z) \in \mathbf{C} \backslash\right]-\infty, 0\right]$. Lemma 12 is proved.

It remains to prove assertion (d) of Proposition 3. This is given by the following lemma. 
Lemma 13. Denote

$$
\mathscr{D}_{4}=\left\{z \in \mathbf{C}: \operatorname{Re} z>-\delta_{H} \theta(1+\varepsilon)\right\} .
$$

Then, there exist a constant $C$ depending only on $\theta$ and the index $H$ such that for $T$ large enough and $z \in \mathscr{D}_{4}$

$$
\sqrt{\frac{1}{4}} \leqslant\left|\exp \left(-\widetilde{\widetilde{R}}_{T}(z)\right)\right| \leqslant \sqrt{C\left(1+\frac{1}{T}+\frac{1}{T \varepsilon}\right)} .
$$

P r o of of Le m m a 13. Observe that

$$
\left|\exp \left(-\widetilde{R}_{T}(z)\right)\right|=\sqrt{\frac{\left|\widetilde{R}_{1, T}(z)\right|}{\left(2+p_{H}\right)|z|\left|z+\delta_{H} \theta\right|}}
$$

From (7.7) and (7.8),

$$
\begin{aligned}
\frac{\left|\widetilde{P}_{T}(z)\right|}{\left(2+p_{H}\right)|z|\left|z+\delta_{H} \theta\right|}-\frac{C}{T^{2}} & \leqslant \frac{\left|\widetilde{\widetilde{R}}_{1, T}(z)\right|}{\left(2+p_{H}\right)|z|\left|z+\delta_{H} \theta\right|} \\
& \leqslant \frac{\left|\widetilde{P}_{T}(z)\right|}{\left(2+p_{H}\right)|z|\left|z+\delta_{H} \theta\right|}+\frac{C}{T^{2}} .
\end{aligned}
$$

On the one hand, the very definition of $\widetilde{P}_{T}$ implies that

$$
\frac{\widetilde{P}(z)}{\left(2+p_{H}\right) z\left(z+\delta_{H} \theta\right)}=\frac{1}{z}\left[z+\frac{r_{1}}{T}+\frac{\theta r_{1}\left(1-\delta_{H}\right)}{T(z+\delta \theta)}\right]
$$

and since $r_{1} \theta>0$, we find that for $z \in \mathbf{C}$ such that $\operatorname{Re} z>-\delta_{H} \theta$, and for $T>2 r_{1} \theta^{-1} \delta_{H}^{-1}$

$$
\left|\frac{\widetilde{P}(z)}{\left(2+p_{H}\right) z\left(z+\delta_{H} \theta\right)}\right| \geqslant \frac{1}{|z|} \sqrt{\left[\operatorname{Re} z+\frac{r_{1}}{T}\right]^{2}+(\operatorname{Im} z)^{2}} \geqslant \frac{1}{2} .
$$

On the other hand,

$$
\frac{\widetilde{P}(z)}{\left(2+p_{H}\right) z\left(z+\delta_{H} \theta\right)}=1+\frac{r_{1}}{T} \frac{1}{\left(2+p_{H}\right) z}+\frac{r_{1} \theta\left(1+\delta_{H}\right)}{T} \frac{1}{\left(2+p_{H}\right) z\left(z+\delta_{H} \theta\right)}
$$

and

$$
\left|\frac{\widetilde{P}(z)}{\left(2+p_{H}\right) z\left(z+\delta_{H} \theta\right)}\right| \leqslant 1+\frac{r_{1}}{\theta \delta_{H} T\left(2+p_{H}\right)}+\frac{r_{1} \theta\left(1+\delta_{H}\right)}{T} \frac{1}{\left(2+p_{H}\right) \delta_{H}^{2} \theta^{2} \varepsilon} .
$$

Lemma 13 is proved.

Finally, the proof of Proposition 3 follows from the conjunction of Lemmas 10 to 13 . 
Pr o of of Proposition 4. Using the decomposition (4.9), assertion (c) of Proposition 3, the fact that for $a \in \mathscr{D}_{1} \cap \mathbf{R}$,

$$
0<\inf _{u \in \mathbf{R}} \exp \left(\check{R}_{T}(a+i u)\right) \leqslant \sup _{u \in \mathbf{R}} \exp \left(\check{R}_{T}(a+i u)\right)<\infty
$$

and the equality $\operatorname{Re}(a+i u)=\operatorname{Re} a$, we only have to bound

$$
u \mapsto \exp (T(L(a+i u)-L(a))) .
$$

The bound is clearly given by inequality (7.1).

Pro of of Proposition 5. Recall that, by Lemma 12, $\check{R}_{T}(a)=\widetilde{\widetilde{R}}_{T}(\varphi(a))$ with $\widetilde{\widetilde{R}}_{T}$ given in (7.5). Moreover, there exist $0<x_{T}^{-}<$ $x_{T}^{+}<-\delta_{H} \theta$ such that for all $\left.x \in\right]-\delta_{H},+\infty[$

$$
\widetilde{\widetilde{R}}_{1, T}(x)-\left(2+p_{H}\right)\left(x-x_{T}^{-}\right)\left(x-x_{T}^{+}\right)=\mathscr{O}\left(T^{-2}\right) .
$$

Since $0<x_{T}^{-}<x_{T}^{+}<-\delta_{H} \theta$, we have for all $\left.x \in\right]-\delta_{H},+\infty[$

$$
\left|\frac{\left(2+p_{H}\right)\left(x-x_{T}^{-}\right)\left(x-x_{T}^{+}\right)}{\left(2+p_{H}\right) x\left(x-\delta_{H} \theta\right)}\right| \leqslant 1
$$

Consequently,

$$
\begin{aligned}
\exp \left(-\check{R}_{T}(a)\right) & \leqslant \sqrt{1+\frac{\widetilde{R}_{1, T}(\varphi(a))-\left(2+p_{H}\right)\left(\varphi(a)-x_{T}^{-}\right)\left(\varphi(a)-x_{T}^{+}\right)}{\left(2+p_{H}\right) \varphi(a)\left(\varphi(a)+\delta_{H} \theta\right)}} \\
& =\mathscr{O}\left(\max \left\{1 ; \frac{-1}{T\left(\varphi(a)+\delta_{H} \theta\right)}\right\}\right)
\end{aligned}
$$

which ends the proof of Proposition 5.

7.3. A contour integral for the Gamma function. In order to obtain an asymptotic expansion for $B_{T}$, it is necessary to make use of the following lemma which slightly extends Lemma 7.3 of [3]. First of all, let $f_{a, b}$ be the density function of the gamma $\mathscr{G}(a, b)$ distribution with parameters $a, b>0$, given by

$$
f_{a, b}(x)= \begin{cases}\frac{b^{a}}{\Gamma(a)} x^{a-1} \exp (-b x) & \text { if } x>0 \\ 0 & \text { otherwise. }\end{cases}
$$

For all integers $k, \ell \geqslant 0$, and for all positive real numbers $\sigma^{2}, \gamma, \nu$, let

$$
v_{k}(a, b, \ell)=\frac{2 \pi \sigma^{2 k} i^{\ell}}{2^{k} k ! \gamma^{2 k+\ell+1}} f_{a, b}^{(2 k+\ell)}(1) .
$$


Lemma 14. For any integers $p>0$ and $\ell \geqslant 0$, we have

$$
\int_{\mathbf{R}} \exp \left(-i \gamma u-\frac{\sigma^{2} u^{2}}{2 T}\right) \frac{u^{\ell}}{(1-2 i \nu u)^{a}} d u=\sum_{k=0}^{p} \frac{v_{k}(a, b, \ell)}{T^{k}}+\mathscr{O}\left(\frac{1}{T^{p+1}}\right)
$$

with $b=\gamma /(2 \nu)$.

P r o of of L e m m a 14. Denote by $N_{\sigma}$ the Gaussian kernel with the positive variance $\sigma^{2}$ :

$$
N_{\sigma}(x)=\frac{1}{\sigma \sqrt{2 \pi}} \exp \left(-\frac{x^{2}}{2 \sigma^{2}}\right)
$$

It is well known that the characteristic functions of $N_{\sigma}$ and $f_{a, b}$ are given by

$$
\widehat{N}_{\sigma}(x)=\exp \left(-\frac{\sigma^{2} x^{2}}{2}\right) \quad \text { and } \quad \hat{f}_{a, b}(x)=\left(1-\frac{i x}{b}\right)^{-a},
$$

respectively. Then, it follows from $[17$, p. 177] that for all integer $\ell \geqslant 0$ and for all positive real numbers $a, b, \tau$

$$
\int_{\mathbf{R}} \exp \left(-i v x-\frac{\tau^{2} v^{2}}{2}\right) v^{\ell} \hat{f}_{a, b}(v) d v=2 \pi i^{\ell} f_{a, b} * N_{\tau}^{(\ell)}(x) .
$$

Via the same lines as in [3, Section 7.3], it is not hard to see that for any $p>0$

$$
f_{a, b} * N_{\tau}^{(\ell)}(x)=\sum_{k=0}^{p} \frac{\tau^{2 k}}{2^{k} k !} f_{a, b}^{(2 k+\ell)}(x)+\mathscr{O}\left(\tau^{2(p+1)}\right) .
$$

Hence, we deduce from the conjunction of (7.11) and (7.12) with $\tau^{2}=$ $\sigma^{2} /\left(T \gamma^{2}\right)$ that

$$
\begin{aligned}
\int_{\mathbf{R}} \exp \left(-i v x-\frac{\sigma^{2} v^{2}}{2 T \gamma^{2}}\right) v^{\ell} \hat{f}_{a, b}(v) d v & \\
& =2 \pi i^{\ell} \sum_{k=0}^{p} \frac{\sigma^{2 k}}{2^{k} k ! \gamma^{2 k} T^{k}} f_{a, b}^{(2 k+\ell)}(x)+\mathscr{O}\left(\frac{1}{T^{p+1}}\right) .
\end{aligned}
$$

Finally, by taking the values $x=1$ and $b=\gamma /(2 \nu)$ together with the change of variables $u=v / \gamma$ in (7.13), we find that

$$
\int_{\mathbf{R}} \exp \left(-i \gamma u-\frac{\sigma^{2} u^{2}}{2 T}\right) \frac{u^{\ell}}{(1-2 i \nu u)^{a}} d u=\sum_{k=0}^{p} \frac{v_{k}(a, b, \ell)}{T^{k}}+\mathscr{O}\left(\frac{1}{T^{p+1}}\right),
$$

which completes the proof of Lemma 14.

Acknowledgments. The authors thank Marina Kleptsyna for very fruitful discussions on Laplace transforms of functionals of fractional Brownian motion. 


\section{REFERENCES}

1. Bahadur R. R., Ranga Rao R. On deviations of the sample mean. - Ann. Math. Statist., 1960, v. 31, p. 1015-1027.

2. Bercu B., Gamboa F., Rouault A. Large deviations for quadratic forms od stationary Gaussian processes. - Stochastic Process. Appl., 1997, v. 71, № 1, p. 75-90.

3. Bercu B., Rouault A. Sharp large deviations for the Ornstein-Uhlenbeck process. Теория вероятн. и ее примен., 2001, т. 46, в. 1, с. 74-93.

4. Bishwall J. P. N. Large deviations in testing fractional Ornstein-Uhlenbeck models. Statist. Probab. Lett., 2008, v. 78, № 8, p. 953-962.

5. Brouste A., Kleptsyna M. Asymptotic properties of MLE for partially observed fractional diffusion system. - Stat. Inference Stoch. Process., 2010, v. 13, № 1, p. 1-13.

6. Bryc W., Dembo A. Large deviations for quadratic functionals of Gaussian processes. - J. Theoret. Probab., 1997, v. 10, № 2, p. 307-332.

7. Dembo A., Zeitouni O. Large Deviations Techniques and Applications. New York: Springer-Verlag, 1998, 396 p.

8. Florens-Landais D., Pham H. Large deviations in estimation of an OrnsteinUhlenbeck model. - J. Appl. Probab., 1999, v. 36, № 1, p. 60-77.

9. Gamboa F., Rouault A., Zani M. A functional large deviations principle for quadratic forms of Gaussian stationary processes. - Statist. Probab. Lett., 1999, v. 43, № 3, p. 299-308

10. Hu Y., Nualart D. Parameter estimation for fractional Ornstein-Uhlenbeck processes. - Statist. Probab. Lett., 2010, v. 80, № 11-12, p. 1030-1038.

11. Jost C. Transformation formulas for fractional Brownian motion. - Stochastic Process. Appl., 2006, v. 116, № 10, p. 1341-1357.

12. Kleptsyna M.L., Le Breton A. Statictical analysis of the fractional OrnsteinUhlenbeck type process. - Stat. Inference Stoch. Process., 2002, v. 5, № 3, p. 229-248.

13. Лебедев Н. Н. Специальные функции и их приложения. М.-Л.: Физматгиз, 1963, $358 \mathrm{c}$.

14. Norros I., Valkeila E., Virtamo J. An elementary approach to a Girsanov formula and other analytical results on fractional Brownian motion. - Bernoulli, 1999, v. 5, № 4, p. 571-587.

15. Prakasa Rao B. L.S. Sequential estimation for fractional Ornstein-Uhlenbeck type process. - Sequential Anal., 2004, v. 23, № 1, p. 33-44.

16. Prakasa Rao B. L. S. Estimation for translation of a process driven by fractional Brownian motion. - Stoch. Anal. Appl., 2005, v. 23, №6, p. 1199-1212.

17. Rudin W. Real and Complex Analysis. New York: McGraw-Hill, 1987, 416 p.

Поступила в редакцию 25.V.2009

Исправленный вариант

14.IX.2010 